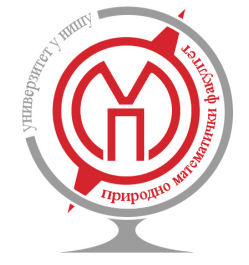

\title{
Completeness Types for Uniformity Theory on Textures
}

\author{
Filiz Y1ld1z \\ ${ }^{a}$ Department of Mathematics, Hacettepe University, Ankara, Turkey
}

\begin{abstract}
.
In this paper the author considers the various types of completeness for di-uniform texture spaces and especially for complemented ones. Following that, the relationships between completeness of uniform spaces and these types of completeness for complemented di-uniform texture spaces are investigated in a categorical setting, just as interrelations between quasi-uniform spaces and di-uniform texture spaces are pointed out insofar as completeness is concerned. Additionally, useful requirements among the various types of completeness of a di-uniformity and real dicompactness of the uniform ditopological space generated by that di-uniformity are presented as a diagram.
\end{abstract}

\section{Introduction and Preliminaries}

As will be clear from the general standard references [3-6], it is showed that ditopological texture spaces provide a unified setting for the study of topology, bitopology and fuzzy topology on Hutton algebras. Some of the links with Hutton spaces and fuzzy topologies are expressed in a categorical setting in [28]. We will not be interested in the links with fuzzy topology in this paper. On the other hand, there is a close relationship between bitopological and ditopological spaces as shown in [22-28].

The foundations of a suitable uniformity theory on textures giving descriptions in terms of direlations, dicovers and dimetrics have been developed in [16] and the term di-uniformity was introduced to cover both dicovering and direlational uniformities. In this work, constant reference will be made to [16] for definitions and results relating to di-uniformities, most of which will be repeated here. Following this, the relationships between quasi-uniformities and uniformities (see [10]) on a set in the classical sense are then investigated in [17], in the setting of di-uniformities on a special texture.

On the other hand, the subjects of completeness and total boundedness for di-uniformities are discussed in [19] and the term dicompleteness is defined as a type of completeness for di-uniform texture spaces. In addition, [18] gives a categorical point of view for the di-uniform texture spaces by defining various categories and functors.

Motivation and background material specific to the main topic of this paper maybe found in [12], [1428]. Due to lack of space, most of this material is not repeated here. In particular, the reader is referred to

2010 Mathematics Subject Classification. Primary 54E15, 54E50, 54B30, 54D35, 18A22 ; Secondary 54C30, 06D10, $03 E 20$.

Keywords. Quasi-Uniformity; Realcompact; Cauchy filter pair; Quotient Category; Complementation; Bicomplete; B-Complete; Jointly Complete; Dicomplete; Texture; C-space; Ditopology; Real Dicompact; Di-Uniformity; Difilter; Plain Dicompletion.

Received: 16 October 2014; Accepted: 18 December 2014

Communicated by Dragan Djurčić

The author acknowledges support under research Project No.014 G 602 001, awarded by the Hacettepe University

Email address: yfiliz@hacet tepe.edu.tr (Filiz Yıldız) 
$[8,9]$ for a more categorically based treatment of completeness and bitopological realcompactness which is the bitopological counterpart of classical realcompactness defined in [12].

In addition, the reader may consult [11] for terms from lattice theory not mentioned here. Also our standard reference for notions and results from category theory is [1] and if $\mathbf{A}$ is a category, $\mathrm{Ob} \mathbf{A}$ will denote the class of objects and Mor $\mathbf{A}$ the class of morphisms of $\mathbf{A}$.

In the present paper, a detailed analysis of the relationships among several certain types of completeness in the various categories of di-uniform texture spaces and the completeness of (quasi-)uniform spaces will be given. Accordingly, the layout of paper is as follows:

After presenting some preliminary material required for the paper, in Section 2 mainly, we introduce the category ifDiU of di-uniform texture spaces and the uniformly bicontinuous point functions satisfying a compatible condition. Following this, Section 3 considers the relationships between quasi-uniform spaces and the corresponding elements of category ifDiU in a categorical setting insofar as completeness is concerned.

Besides, in Section 4 after recalling the notions of complementation, complemented texture and the other concepts related with a complementation, the effect of a complementation on the di-uniformities is taken up. Accordingly, the subcategory ifCDiU of ifDiU whose objects are complemented di-uniform texture spaces, is established and a type of completeness peculiar to the category ifCDiU is introduced by defining a notion of complemented difilter. This section also contains another several definitions and results that are important in their own right, and which will also be needed later on.

Finally, Section 5 as the last part of paper, discusses the connections between the classical uniform spaces and the corresponding elements of category ifCDiU in a categorical setting insofar as completeness is concerned. As a result of these connections, by defining the various types of completeness for di-uniform texture spaces we proved some useful ditopological requirements which contain these types of completeness as well as the notion of real dicompactness. Following that, we conclude the paper by giving a diagram illustrates all those requirements.

Now we end this section by recalling some preliminary information and results that will enable the casual reader to follow the general ideas presented here.

Textures If $S$ is a set, a texturing $\mathcal{S}$ of $S$ is a subset of $\mathcal{P}(S)$ which is a point-separating, complete, completely distributive lattice containing $S$ and $\emptyset$, and for which meet coincides with intersection and finite joins with union. The pair $(S, S)$ is then called a texture.

We regard a texture as a framework in which to do mathematics.

For a texture $(S, \mathcal{S})$, most properties are conveniently defined in terms of the $p$-sets and $q$-sets:

$$
P_{s}=\bigcap\{A \in \mathcal{S} \mid s \in A\}, \quad Q_{s}=\bigvee\{A \in \mathcal{S} \mid s \notin A\}
$$

On the other hand, as noted in [3] we may associate with $(S, S)$ the C-space $\left(S, S^{c}\right)$ that is, a $T_{0}$ topological space with a completely distributive lattice of open sets, and then the frequently occurring relationship $P_{s^{\prime}} \nsubseteq Q_{s}, s, s^{\prime} \in S$, is equivalent to $s \omega_{S} s^{\prime}$, where $\omega_{S}$ is the interior relation for the $C$-space $\left(S, \delta^{c}\right)$.

Ditopological Texture Spaces Since a texturing $\mathcal{S}$ need not be closed under the operation of taking the set-complement, the notion of topology is replaced by that of dichotomous topology or ditopology, namely a pair $(\tau, \kappa)$ of subsets of $\mathcal{S}$, consists of the set $\tau$ of open sets and the set $\kappa$ of closed sets satisfying some conditions. Hence a ditopology is essentially a "topology" for which there is no a priori relation between the open and closed sets. A ditopological texture space with respect to a ditopology $(\tau, \kappa)$ on the texture $(S, \mathcal{S})$ is denoted by $(S, \mathcal{S}, \tau, \kappa)$. For details see [5].

Functions Between Textures The notions of direlation and difunction between textures are considered in detail in [5] and the category dfTex of textures and difunctions is introduced.

A point function $\varphi$ between the base sets of two textures $(S, S),(T, \mathcal{T})$ that preserves the interior relations, that is satisfying the condition $s \omega_{S} s^{\prime} \Longrightarrow \varphi(s) \omega_{T} \varphi\left(s^{\prime}\right)$, is called $\omega$-preserving. Hence, the category of 
textures and $\omega$-preserving point functions is denoted by ifTex. This category which has products was introduced in [21] and some subcategories of it were defined in [20,21, 24, 25]. Also, a major part of the theory of point functions may be found in [23, 24].

In general difunctions are not directly related to ordinary (point) functions between the base sets, but we recall from [5, Lemma 3.4] that if $(S, \mathcal{S}),(T, \mathcal{T})$ are textures and $\varphi: S \rightarrow T$ a point function satisfying the compatibility condition $P_{s} \nsubseteq Q_{s^{\prime}} \Longrightarrow P_{\varphi(s)} \nsubseteq Q_{\varphi\left(s^{\prime}\right)}$, then the formulae

$$
\begin{aligned}
& f_{\varphi}=\bigvee\left\{\bar{P}_{(s, t)} \mid \exists u \in S \text { with } P_{S} \nsubseteq Q_{u} \text { and } P_{\varphi(u)} \nsubseteq Q_{t}\right\}, \\
& F_{\varphi}=\bigcap\left\{\bar{Q}_{(s, t)} \mid \exists v \in S \text { with } P_{v} \nsubseteq Q_{s} \text { and } P_{t} \nsubseteq Q_{\varphi(v)}\right\},
\end{aligned}
$$

define a difunction $\left(f_{\varphi}, F_{\varphi}\right)$ from $(S, \mathcal{S})$ to $(T, \mathcal{T})$.

Also the following equalities define the inverse image with respect to $\varphi$ and $\left(f_{\varphi}, F_{\varphi}\right)$

$$
f_{\varphi}^{\leftarrow B}=F_{\varphi}^{\leftarrow} B=\varphi^{\leftarrow} B=\bigvee\left\{P_{u} \mid \varphi(u) \in B\right\}=\bigcap\left\{Q_{v} \mid \varphi(v) \notin B\right\} .
$$

for each $B \in \mathcal{T}$.

Bicontinuity A difunction or an $\omega$-preserving point function between the ditopological texture spaces, is called bicontinuous if the inverse image of every open set is open and the inverse image of every closed set is closed.

Special Classes of Textures The texture $(S, \mathcal{S})$ is called plain if $\mathcal{S}$ is closed under arbitrary unions.

The more general class of nearly plain textures and the notion of $*$-space, required for the rest of paper, were introduced in [24]. Recall that a texture $(S, S)$ is called nearly plain if given $s \in S$ there exists a point $a \in S$ satisfying $Q_{s}=Q_{a}$ for which $a \omega_{S} a$. Clearly every ditopological plain texture space is nearly plain and *-space.

In this work, the set of plain points of a nearly plain texture $(S, \delta)$ is denoted by $S_{p}=\left\{s \in S \mid s \omega_{S} s\right\}$ such as in the papers [21-28].

Also the following notion, which is given in [26] and weaker than that of nearly plain texture, is required for the rest of paper:

Given $s_{1}, s_{2} \in S$ with $s_{1} \omega_{S} s_{2}$ if there exists $u \in S_{p}$ with $s_{1} \omega_{S} u$ and $u \omega_{S} s_{2}$ then the texture $(S, S)$ is called almost plain.

In this work, the subconstruct of ifTex whose objects are plain is denoted by ifPTex. Recall from [20], ifPTex is a full, isomorphism-closed, concretely reflective subconstruct of ifTex.

Note that ifPTex coincides with the category fPTex given in [5] and we recall that fPTex is isomorphic to dfPTex, which is defined in [5], and consisting of plain textures and dfTex-morphisms. Consequently, ifPTex is isomorphic to dfPTex.

We denote by ifDitop (see [21]) the category of ditopological texture spaces and bicontinuous ifTexmorphisms. Clearly ifDitop is topological category over ifTex. In addition, the following proposition which is given in [21] with its proof, will be required for the rest of paper.

Proposition 1.1. An ifTex morphism $\varphi: S \rightarrow T$ is an ifTex-isomorphism if and only if

i) $\varphi$ is bijective,

ii) $P_{s} \subseteq Q_{u}$ then $P_{\varphi(s)} \subseteq Q_{\varphi(u)}$.

Similar to Proposition 1.1, ifDitop-isomorphisms are characterized in [21]. Hence, it can be seen that an ifDitop-isomorphism is in fact a bicontinuous ifTex-isomorphism such that its inverse is also bicontinuous.

For the benefit of reader, let us give three useful examples: 
Examples 1.2. (1) For any set $X,(X, \mathcal{P}(X))$ is the discrete texture representing the usual set structure of $X$. Clearly, $P_{x}=\{x\}, Q_{x}=X \backslash\{x\}$ for all $x \in X$. Hence, $(X, \mathcal{P}(X)) \in$ Ob ifPTex. Also for any topology $\mathcal{T}$ on $X$, $\left(\mathcal{T}, \mathcal{T}^{c}\right)$ is a ditopology on $(X, \mathcal{P}(X))$ where $\mathcal{T}^{c}=\{X \backslash G \mid G \in \mathcal{T}\}$.

Moreover, for any bitopology $(u, v)$ on $X,\left(u, v^{c}\right)$ is a ditopology on $(X, \mathcal{P}(X))$.

(2) Consider the real texture $(\mathbb{R}, \mathcal{R})$. Here $\mathbb{R}$ denotes the set of real numbers and $\mathcal{R}$ is the texturing $\{(-\infty, r] \mid r \in$ $\mathbb{R}\} \cup\{(-\infty, r) \mid r \in \mathbb{R}\} \cup\{\mathbb{R}, \emptyset\}$. Here $P_{r}=(-\infty, r]$ and $Q_{r}=(-\infty, r)$ for all $r \in \mathbb{R}$, thus $(\mathbb{R}, \mathcal{R}) \in$ Ob ifPTex. In addition, the natural ditopology on $(\mathbb{R}, \mathcal{R})$ is $\left(\tau_{\mathbb{R}}, \kappa_{\mathbb{R}}\right)$, where $\tau_{\mathbb{R}}=\{(-\infty, r) \mid r \in \mathbb{R}\} \cup\{\mathbb{R}, \emptyset\}$ and $\kappa_{\mathbb{R}}=$ $\{(-\infty, r] \mid r \in \mathbb{R}\} \cup\{\mathbb{R}, \emptyset\}$. Thus real ditopological texture space is denoted by $\left(\mathbb{R}, \mathcal{R}, \tau_{\mathbb{R}}, \kappa_{\mathbb{R}}\right)$ in this context.

(3) For $\mathbb{I}=[0,1]$ define $\mathcal{J}=\{[0, t] \mid t \in[0,1]\} \cup\{[0, t) \mid t \in[0,1]\}$. We will refer to $(\mathbb{I}, \mathcal{J})$ as the unit interval texture. Here $P_{t}=[0, t]$ and $Q_{t}=[0, t)$ for all $t \in \mathbb{I}$ and $(\mathbb{I}, \mathcal{J}) \in$ Ob ifPTex. Also the equalities $\tau_{\mathbb{I}}=\{[0, r) \mid 0 \leq r \leq 1\} \cup\{\mathbb{I}\}, \kappa_{\mathbb{I}}=\{[0, r] \mid 0 \leq r \leq 1\} \cup\{\emptyset\}$ defines a ditopology, called the natural ditopology on $(\mathbb{I}, \mathcal{J})$. Thus the notation $\left(\mathbb{I}, \mathcal{J}, \tau_{\mathbb{I}}, \kappa_{\mathbb{I}}\right)$ will denote the ditopological unit interval texture space.

Now let us recall the other required notions for the present paper:

$T$-Lattices of Real (Di)Functions Unlike the classical case given in [12], the families $\operatorname{BDF}(S)=\{(f, F) \mid(f, F)$ : $(S, \mathcal{S}, \tau, \mathcal{\kappa}) \rightarrow\left(\mathbb{R}, \mathcal{R}, \tau_{\mathbb{R}}, \kappa_{\mathbb{R}}\right)$ is bicontinuous difunction $\}$ and $\mathrm{BA}(S)=\left\{\varphi \mid \varphi:(S, \mathcal{S}, \tau, \kappa) \rightarrow\left(\mathbb{R}, \mathcal{R}, \tau_{\mathbb{R}}, \kappa_{\mathbb{R}}\right)\right.$ is $\omega$-preserving and bicontinuous point function\} given in [24], are not rings but $T$-lattices. Such parts of the theory of $T$-lattice developed in [2] and required here, have already been given in [22, 24-26], and will not be repeated.

Separation Axioms in Textures The basic separation axioms for ditopological texture spaces are discussed in [7] and the reader is referred to it for the definitions of $T_{0}$, complete bi-regularity and bi- $T_{2}$ which effectively represents the Hausdorff axiom in this context, also to [24, Proposition 1.1] for a characterization of complete biregularity suitable for our present purposes.

Real Dicompact Texture Spaces Based on the foundation laid in [24], the author presents in [25] a suitable realcompactness structure for ditopological texture spaces, under the name real dicompactness as a natural counterpart of the classical notion of realcompactness defined in [12], also proves that the real ditopological space $\left(\mathbb{R}, \mathcal{R}, \tau_{\mathbb{R}}, \kappa_{\mathbb{R}}\right)$ given as Examples $1.2(2)$, is real dicompact.

Dicovers: A set $\mathcal{D} \subseteq \mathcal{S} \times \mathcal{S}$ is called a difamily on the texture $(S, \mathcal{S})$ and a difamily $\mathcal{C}=\left\{\left(A_{j}, B_{j}\right) \mid j \in J\right\}$ of elements of $\mathcal{S} \times \mathcal{S}$ which satisfies $\bigcap_{j \in J_{1}} B_{j} \subseteq \bigvee_{j \in J_{2}} A_{j}$ for all partitions $\left(J_{1}, J_{2}\right)$ of $J$, including the trivial partitions, is called a dicover of $(S, \mathcal{S})$. This notion defined in [4] is the textural counterpart of the notion dual cover introduced in [2].

Uniformity on Textures : Just as uniformities can be described by using covers in the classical case so a suitable uniformity theory on textures can be described in terms of dicovers and the resulting structure is called dicovering uniformity, defined in [16, Definition 3.6]. In particular, the same uniformity theory on textures can be described in terms of direlations as well and the term direlational uniformity is used for direlational case.

Uniform Ditopology: Recall that just as a uniformity in the classical sense determines a topology called the uniform topology, so a di-uniformity determines a ditopology called the uniform ditopology introduced in [16].

Difilters An appropriate notion of "filter" on a texture would seem to be that of difilter by the notation $\mathcal{F} \times \mathcal{G}$, defined in [19]. It follows from the definition that the difilters are very general, so we imposes a suitable "regularity" condition on them. The related details about (regular) difilters are given in [19, 21, 24].

Dicomplete Texture Spaces: The subjects of completeness and total boundedness for di-uniformities are discussed in [19]. Particularly, the notions of Cauchy difilter and dicompleteness as completeness of di-uniform texture spaces are defined as follows:

Definition 1.3. Let $(S, S)$ be a texture and $v$ a dicovering uniformity on $(S, S)$.

1. A difilter $\mathcal{F} \times \mathcal{G}$ on $(S, \mathcal{S})$ is said to be Cauchy if $(\mathcal{F} \times \mathcal{G}) \cap \mathcal{C} \neq \emptyset$ for all $\mathcal{C} \in v$.

2. $(S, \mathcal{S}, v)$ is called dicomplete if every regular Cauchy difilter is diconvergent. 
Here note that diconvergency of difilters on a texture will be with respect to the uniform ditopology. For another details about dicompleteness see [27].

In addition, a certain type of completion for di-uniformities on an almost plain texture is constructed under the name plain dicompletion in [27]. Particularly, [27] associates the completeness of a certain diuniform texture space with the real dicompactness of compatible ditopological texture space and we continue to work within the same framework in the final section of the present paper.

\section{The Category ifDiU}

In this section, we will introduce the category of di-uniform texture spaces and $\omega$-preserving, uniformly bicontinuous point functions, and will consider some related categories.

Firstly, recall from [18, Definition 4.4] that for direlational uniformities $U$ and $V$, an $\omega$-preserving point function $\varphi:(S, \mathcal{S}, \mathcal{U}) \rightarrow(T, \mathcal{T}, \mathcal{V})$ is called $\mathcal{U}-\mathcal{V}$ (direlational) uniformly bicontinuous if $(r, R) \in \mathcal{V}$ then $\varphi^{-1}(r, R) \in \mathcal{U}$.

In a similar way, we will define the notion of dicovering uniform bicontinuity for an $\omega$-preserving point function. Before it, we need to mention what we mean by the inverse image of a dicover under an $\omega$-preserving point function.

For this, let $\varphi: S \rightarrow T$ be an $\omega$-preserving point function and $\mathcal{C}$ a dicover on $(T, \mathcal{T})$ then the inverse image of $\mathcal{C}$ under $\varphi$ is defined as:

$$
\varphi^{-1} \mathcal{C}=\left\{\left(\varphi^{\leftarrow} A, \varphi^{\leftarrow} B\right) \mid A \mathcal{C} B\right\}
$$

Clearly the difamily $\varphi^{-1} \mathcal{C}$ is a dicover since $\mathcal{C}$ is dicover and the delta refinement [16] of $\varphi^{-1} \mathcal{C}$ is defined as follows:

$$
\left(\varphi^{-1} \mathcal{C}\right)^{\Delta}=\left\{\left(\operatorname{St}\left(\varphi^{-1} \mathcal{C}, P_{s}\right), \operatorname{CSt}\left(\varphi^{-1} \mathcal{C}, Q_{s}\right)\right) \mid s \in S^{b}\right\}
$$

where $\operatorname{St}\left(\varphi^{-1} \mathcal{C}, P_{s}\right)=\bigvee\left\{\varphi^{\leftarrow} A_{i} \mid i \in I, P_{s} \nsubseteq \varphi^{\leftarrow} B_{i}\right\} \in \mathcal{S}, \operatorname{CSt}\left(\varphi^{-1} \mathcal{C}, Q_{s}\right)=\bigcap\left\{\varphi^{\leftarrow} B_{i} \mid i \in I, \varphi^{\leftarrow} A_{i} \nsubseteq Q_{s}\right\} \in \mathcal{S}$, $\mathcal{C}=\left\{\left(A_{i}, B_{i}\right) \mid i \in I\right\}$ and $S^{b}=\left\{s \mid S \neq Q_{s}\right\}$.

Now, we will present some propositions and results required for the rest of paper. Firstly, if we recall the equality $\left(f_{\varphi}, F_{\varphi}\right)^{-1} \mathcal{C}=\left\{\left(F_{\varphi}^{\leftarrow} A, f_{\varphi}^{\leftarrow} B\right) \mid A \mathcal{C} B\right\}$ by [16], the following will be obvious:

Proposition 2.1. Let $(S, \mathcal{S})$ and $(T, \mathcal{T})$ be textures, $\varphi: S \rightarrow T$ an $\omega$-preserving point function and $\left(f_{\varphi}, F_{\varphi}\right):(S, \mathcal{S}) \rightarrow$ $(T, \mathcal{T})$ the corresponding difunction. Then the equality $\varphi^{-1} \mathrm{C}=\left(f_{\varphi}, F_{\varphi}\right)^{-1} \mathrm{C}$ is satisfied for a dicover $\mathcal{C}$ on $(T, \mathcal{T})$. have:

Hence, by Proposition 2.1 and the definition of dicovering uniform bicontinuity for a difunction, we

Proposition 2.2. Suppose that $(S, \mathcal{S}, \mathcal{U})$ and $(T, \mathcal{T}, \mathcal{V})$ are dicovering uniform texture spaces, $\varphi: S \rightarrow T$ an $\omega$ preserving point function, and $\left(f_{\varphi}, F_{\varphi}\right):(S, \mathcal{S}) \rightarrow(T, \mathcal{T})$ the corresponding difunction. In this case, $\left(f_{\varphi}, F_{\varphi}\right)$ is dicovering uniformly bicontinuous if and only if $\mathcal{C} \in \mathcal{V} \Longrightarrow\left(\varphi^{-1} \mathcal{C}\right)^{\Delta} \in \mathcal{U}$.

According to the above statements, the following definition can be given:

Definition 2.3. Let $(S, \mathcal{S}, \mathcal{U}),(T, \mathcal{T}, \mathcal{V})$ be dicovering uniform texture spaces and $\varphi: S \rightarrow T$ an $\omega$-preserving point function. If $\mathrm{C} \in \mathcal{V} \Longrightarrow\left(\varphi^{-1} \mathcal{C}\right)^{\Delta} \in \mathcal{U}$ then $\varphi$ is called $\mathcal{U}-\mathcal{V}$ dicovering uniformly bicontinuous.

Thus we have:

Corollary 2.4. Let $(S, \mathcal{S}, \mathcal{U}),(T, \mathcal{T}, \mathcal{V})$ be direlational uniform texture spaces and $\varphi: S \rightarrow T$ an $\omega$-preserving point function. In this case, $\varphi$ is direlational uniformly bicontinuous if and only if $\varphi$ is dicovering uniformly bicontinuous.

Proof. As we can associate a dicovering uniformity with a given direlational uniformity by [16, Theorem 3.7] which expresses the equivalence of these, the proof is trivial from Definition 2.3. 
Note 2.5. On account of Corollary 2.4, in what follows we will use the term uniform bicontinuity for an w-preserving point function to refer to dicovering uniform bicontinuity and direlational uniform bicontinuity, in general.

Therefore, by Proposition 2.2 and Definition 2.3 we have:

Corollary 2.6. An $\omega$-preserving point function $\varphi:(S, \mathcal{S}, \mathcal{U}) \rightarrow(T, \mathcal{T}, \mathcal{V})$ is uniformly bicontinuous if and only if the corresponding difunction $\left(f_{\varphi}, F_{\varphi}\right):(S, \mathcal{S}, \mathcal{U}) \rightarrow(T, \mathcal{T}, \mathcal{V})$ is uniformly bicontinuous.

It is clear that if $\varphi$ satisfies the condition $\mathcal{C} \in \mathcal{V} \Longrightarrow \varphi^{-1} \mathcal{C} \in \mathcal{U}$ then $\varphi$ is uniformly bicontinuous, since $\varphi^{-1} \mathcal{C} \in \mathcal{U}$ implies that $\left(\varphi^{-1} \mathcal{C}\right)^{\Delta} \in \mathcal{U}$.

On the contrary, we have the following:

Proposition 2.7. Suppose that $(S, \mathcal{S}, \mathcal{U})$ is a dicovering uniform texture space and $(T, \mathcal{T}, \mathcal{V})$ a dicovering uniform plain texture space. If $\varphi: S \rightarrow T$ is an $\omega$-preserving point function then the following are equivalent:

i) $\varphi$ is uniformly bicontinuous.

ii) $\mathcal{e} \in \mathcal{V} \Longrightarrow \varphi^{-1} \mathcal{C} \in \mathcal{U}$.

iii) $\mathcal{C} \in \mathcal{V} \Longrightarrow\left(f_{\varphi}, F_{\varphi}\right)^{-1} \mathcal{C} \in \mathcal{U}$

iv) $\left(f_{\varphi}, F_{\varphi}\right)$ is uniformly bicontinuous.

Proof. i) $\Rightarrow$ ii) is clear from [27, Lemma 2.14], ii) $\Rightarrow$ iii) is seen by using Proposition 2.1 and iv) $\Rightarrow$ i) is proved by Corollary 2.6. In addition, $i i i) \Rightarrow i v)$ is obvious from the definition of uniform bicontinuity for a difunction and the fact that $\left(f_{\varphi}, F_{\varphi}\right)^{-1} \mathcal{C} \in \mathcal{U}$ implies $\left(\left(f_{\varphi}, F_{\varphi}\right)^{-1} \mathcal{C}\right)^{\Delta} \in \mathcal{U}$.

Now let us give some considerations in order to describe a category of di-uniform texture spaces:

First of all, we can define the category of direlational uniformities and uniformly bicontinuous ifTexmorphisms since the uniform bicontinuity with respect to direlational uniformities is preserved under composition of $\omega$-preserving point-functions and the identity point function is $\omega$-preserving, uniformly bicontinuous, moreover, it is identity for composition. We will denote this category by $\mathbf{D}$.

Likewise, dicovering uniformities and uniformly bicontinuous ifTex-morphisms form a category. Indeed, if the $\omega$-preserving point functions $\varphi, \psi$ are dicovering bicontinuous then they are direlational bicontinuous by Corollary 2.4, so $\varphi \circ \psi$ is direlational bicontinuous by the definition of composition for point functions and finally, $\omega$-preserving composite point function $\varphi \circ \psi$ is dicovering bicontinuous. We will denote this category by $\mathbf{E}$.

If we take the notations $U: \mathbf{D} \rightarrow$ ifTex, $V: \mathbf{E} \rightarrow$ ifTex denoting obvious forgetful functors then the categories $(\mathbf{D}, U)$ and $(\mathbf{E}, V)$ are concrete over ifTex. Therefore we have:

Theorem 2.8. $(\mathbf{D}, U)$ and $(\mathbf{E}, V)$ are concretely isomorphic categories.

Proof. With the above facts, the proof is trivial by using Corollary 2.4 and [16, Proposition 5.20, Theorem 3.7].

As a result of Theorem 2.8, we will use the term di-uniformity to refer to both direlational and dicovering uniformities on a texture.

We are now in a position to define the category ifDiU whose objects are di-uniform texture spaces and morphisms are $\omega$-preserving, uniformly bicontinuous point functions.

It is clear that ifDiU is concrete category over ifTex with respect to the forgetful functor which we will denote by $\mathfrak{M}:$ ifDiU $\rightarrow$ ifTex defined as $(S, \mathcal{S}, \mathcal{U}) \mapsto(S, S)$. Now we have the following:

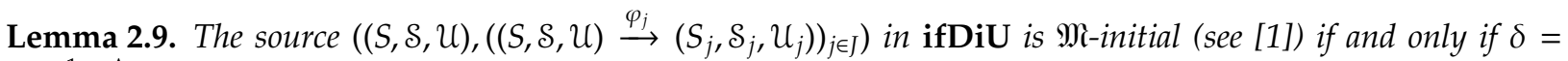
$\left\{\left(\varphi_{j}^{-1} \mathcal{C}\right)^{\Delta} \mid \mathcal{C} \in \mathcal{U}_{j}, j \in J\right\}$ is a subbase for the di-uniformity $\mathcal{U}$. In other words, the di-uniformity $\mathcal{U}$ generated by $\delta$, is the coarsest dicovering uniformity on $(S, S)$ for which the w-preserving point functions $\varphi_{j}, j \in J$, are uniformly bicontinuous. 
Proof. It is similar to the proof of [18, Lemma 2.4].

Hence, the next theorem is clear from Lemma 2.9 by using the notions of topological functor and topological category given in [1].

Theorem 2.10. The forgetful functor $\mathfrak{M}:$ ifDiU $\rightarrow$ ifTex is topological. In other words, ifDiU is topological category over ifTex with respect to the functor $\mathfrak{M}$.

Further we have:

Corollary 2.11. ifDiU has products.

Proof. Firstly, note that the product of textures is also a texture by the definition of "product texturing" given in [5]. Following that, in like manner to the proof of [6, Theorem 3.10] by using the projection point functions (instead of projection difunctions), $\rho_{j}: S=\prod_{j \in J} S_{j} \rightarrow S_{j}(j \in J)$ which are $w$-preserving, it can be seen that ifTex has products. In addition, by [1] it is known that a topological functor preserves limits, thus the required is clear from Theorem 2.10.

Now we will consider the interrelations of the category ifDiU and some of its subcategories with the other known categories peculiar to the texture theory. We begin by noting that just as the category Uni of uniformities and uniformly continuous functions is related to the construct Top of topological spaces and continuous functions via the uniform topology, so the category ifDiU is related to the category ifDitop of ditopological texture spaces and bicontinuous $\omega$-preserving point functions via the uniform ditopology recalled in Section 1.

Specificially, we define 5 : ifDiU $\rightarrow$ ifDitop by

$$
\mathfrak{G}\left(\left(S_{1}, \mathcal{S}_{1}, v_{1}\right) \stackrel{\varphi}{\rightarrow}\left(S_{2}, \mathcal{S}_{2}, v_{2}\right)\right)=\left(S_{1}, \mathcal{S}_{1}, \tau_{v_{1}}, \kappa_{v_{1}}\right) \stackrel{\varphi}{\rightarrow}\left(S_{2}, \mathcal{S}_{2}, \tau_{v_{2}}, \kappa_{v_{2}}\right) .
$$

where $\left(\tau_{v_{i}}, \kappa_{v_{i}}\right)$ is the ditopology generated by the di-uniformity $v_{i}, i=1,2$. Here, $\mathfrak{6}$ is a faithful functor by the expected fact that if $\varphi$ is $v_{1}-v_{2}$ uniformly bicontinuous then it is $\left(\tau_{v_{1}}, \kappa_{v_{1}}\right)-\left(\tau_{v_{2}}, \kappa_{v_{2}}\right)$ bicontinuous.

We know from [16, Theorem 4.14] that the uniform ditopology is always completely biregular. Hence, denoting by ifCbiReg the full subcategory of ifDitop whose objects are completely biregular ditopological texture spaces, we may regard the functor $(\mathfrak{5}:$ ifDiU $\rightarrow$ ifCbiReg as concrete functor over ifTex.

Now let us consider the converse. First of all, note that the following some notations:

For a ditopological texture space $(S, \mathcal{S}, \tau, \mathcal{\kappa})$, the initial di-uniformities (see [27, Section 3$]$ ) generated by the $T$-lattices $\mathrm{BA}(S)=\mathrm{BA}_{\mathbb{R}}(S)$ and $\mathrm{BDF}(S)=\mathrm{BDF}_{\mathbb{R}}(S)$ given in Section 1 , will be denoted by $\mathcal{U}_{\mathrm{BA}(S)}$ and $\mathcal{U}_{\mathrm{BDF}(S)}$ respectively. Here note that $\mathcal{U}_{\mathrm{BA}(S)}$ is coarser than $\mathcal{U}_{\mathrm{BDF}(S)}$ and the equality $\mathcal{U}_{\mathrm{BA}(S)}=\mathcal{U}_{\mathrm{BDF}(S)}$ can be shown easily, for the ditopological nearly plain texture space $(S, S, \tau, \kappa)$. Additionally, for the sub $T$-lattice $\mathrm{BA}^{*}(S)$ of bounded elements of $\mathrm{BA}(S)$, appropriate initial di-uniformity is denoted by $\mathcal{U}_{\mathrm{BA}^{*}(S)}$ and it is clear that $\mathcal{U}_{\mathrm{BA}^{*}(S)}$ is coarser than $\mathcal{U}_{\mathrm{BA}(S)}$.

Following that, it is well known from [16, Theorem 5.16] "The space $(S, S, \tau, \kappa)$ is completely bi-regular if

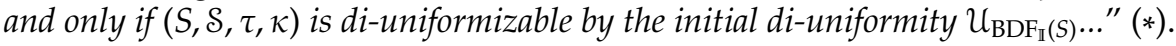

Accordingly, now we will give a theorem corresponding to the above characterization, for the point functions as well.

Theorem 2.12. The ditopological texture space $(S, \mathcal{S}, \tau, \kappa)$ is completely bi-regular if and only if $(S, \mathcal{S}, \tau, \kappa)$ is diuniformizable with the initial di-uniformity $U_{\mathrm{BA}_{\mathrm{I}}(S)}$ (which has the subbase $\delta$ defined in Lemma 2.9) generated by the T-lattice $\mathrm{BA}_{\mathbb{I}}(S)$ of bicontinuous, $\omega$-preserving point functions defined from $S$ to $\mathbb{I}$.

Proof. For the ditopological texture space $(S, S, \tau, \kappa)$, complete bi-regularity was stated in [24, Proposition 1.1] by using the $T$-lattice $\mathrm{BA}_{\mathbb{I}}(S)$ instead of the $T$-lattice $\mathrm{BDF}_{\mathbb{I}}(S)$. In addition, from the above (*) equivalency, complete bi-regularity is characterized by the initial di-uniformity $\mathcal{U}_{\mathrm{BDF}_{\mathrm{I}}(S)}$ in an obvious way. Hence we have the equalities $\tau=\tau_{\mathcal{U}_{\mathrm{BA}_{\mathrm{I}}(S)}}=\tau_{\mathcal{B D D F}_{\mathrm{I}}(S)}$ and $\kappa=\kappa_{\mathcal{U}_{\mathrm{BA}_{\mathrm{I}}(S)}}=\kappa_{\mathcal{U}_{\mathrm{BDF}_{\mathrm{I}}(S)}}$ for the completely bi-regular ditopological texture space $(S, \mathcal{S}, \tau, \kappa)$. 
By virtue of the above theorem, clearly the functor $(55$ is surjective on objects and a section, also it preserves initial sources and it is an adjoint.

On the other hand, if we restrict the objects in the category ifDiU to di-uniformities on textures which are plain, nearly plain and almost plain we obtain the full subcategories ifPDiU, ifNpDiU and ifApDiU of ifDiU, respectively.

Similar to Corollary 2.11, the subcategories ifPDiU, ifNpDiU and ifApDiU have products as well. Because, the product of plain textures is a plain texture from [21, Theorem 2.2.1] and similarly, the product of nearly plain textures is nearly plain texture from [24, Page 177]. Also, the product of almost plain textures is an almost plain texture from [26, Page 3044].

Now we have:

Proposition 2.13. ifPDiU is a full, isomorphism-closed concretely reflective subcategory of ifDiU.

Proof. Fullness is obvious since the morphisms are same. The fact that ifPDiU is isomorphism-closed in ifDiU follows from by [20, Proposition 2.4] and by the statement plainness is preserved under an ifTexisomorphism (see Proposition 1.1). For $(S, \mathcal{S}, \mathcal{U}) \in \mathrm{Ob}$ ifDiU, define $\left(S, \mathcal{L}_{S}\right)$ and the ifTex-morphism $\sigma:(S, \mathcal{S}) \rightarrow$ $\left(S, \mathcal{L}_{S}\right)$ as in the proof of [20, Proposition 2.4]. Thus, it is clear that $\sigma:(S, S) \rightarrow\left(S, \mathcal{L}_{S}\right)$ is an ifPTex-reflection arrow. Also ifPDiU is clearly concrete over ifTex.

Now, let us construct the di-uniformity $\mathcal{V}=\left\{\mathcal{C} \mid\left(\sigma^{\leftarrow} \mathcal{C}\right)^{\Delta} \in \mathcal{U}\right\}$ on $\left(S, \mathcal{L}_{S}\right)$. In this case, if we recall the diagram given in the proof of [20, Proposition 2.4] then it suffices to show that $\varphi^{*}=\varphi: S \rightarrow T$ is $\mathcal{V}-\mathcal{W}$ uniformly bicontinuous, where $\mathcal{W}$ is a di-uniformity on $(T, \mathcal{T}) \in \operatorname{Ob}$ ifPTex and $\varphi:(S, \mathcal{S}, \mathcal{U}) \rightarrow(T, \mathcal{T}, \mathcal{W})$ is uniformly bicontinuous. Now, by virtue of $\varphi=\varphi^{*} \circ \sigma$ we have $\varphi^{\leftarrow} \mathcal{C}=\sigma^{\leftarrow}\left(\left(\varphi^{*}\right)^{\leftarrow} \mathcal{C}\right)$ for $\mathcal{C} \in \mathcal{W}$. Hence, $\varphi^{\leftarrow} \mathcal{C} \in \mathcal{U}$ since $\varphi$ is $\mathfrak{U}-\mathcal{W}$ uniformly bicontinuous. Also, note that as the texture $\left(S, \mathcal{L}_{S}\right)$ is plain, we can use the notation $\sigma^{\leftarrow} \mathcal{C}$ instead of $\left(\sigma^{\leftarrow} \mathcal{C}\right)^{\Delta}$ by Proposition $2.7(i) \Leftrightarrow(i i)$ and so $\left(\varphi^{*}\right)^{\leftarrow} \mathcal{C} \in \mathcal{V}$ by the equality $\mathcal{V}=\{\mathcal{C} \mid \sigma \leftarrow \mathcal{C} \in \mathcal{U}\}$. Finally, $\varphi^{*}$ is $\mathcal{V}-\mathcal{W}$ uniformly bicontinuous and so $\sigma$ is an ifPDiU-reflection arrow.

Practically, the category $\mathbf{d f D i U}$ of di-uniform texture spaces and uniformly bicontinuous difunctions is defined and studied in [18].

Therefore, we define $\mathfrak{D}:$ ifDiU $\rightarrow$ dfDiU by

$$
\mathfrak{D}((S, \mathcal{S}, \mathcal{U}) \stackrel{\varphi}{\rightarrow}(T, \mathcal{T}, \mathcal{V}))=(S, \mathcal{S}, \mathcal{U}) \stackrel{\left(f_{\varphi}, F_{\varphi}\right)}{\longrightarrow}(T, \mathcal{T}, \mathcal{V})
$$

since the $\omega$-preserving, uniformly bicontinuous point function $\varphi$ gives the corresponding uniformly bicontinuous difunction $\left(f_{\varphi}, F_{\varphi}\right)$ by Corollary 2.6.

According to these considerations we have:

Theorem 2.14. $\mathfrak{D}:$ ifDiU $\rightarrow$ dfDiU is a functor. As the restriction to plain textures, the functor $\mathfrak{D}_{p}:$ ifPDiU $\rightarrow$ dfPDiU is an isomorphism with its inverse $\mathfrak{B}_{p}: \mathbf{d f P D i U} \rightarrow \mathbf{i f P D i U}$ given by

$$
\mathfrak{B}_{p}((S, \mathcal{S}, \mathcal{U}) \stackrel{(f, F)}{\longrightarrow}(T, \mathcal{T}, \mathcal{V}))=(S, \mathcal{S}, \mathcal{U}) \stackrel{\varphi_{(f, F)}}{\longrightarrow}(T, \mathcal{T}, \mathcal{V}) .
$$

where $(f, F)=\left(f_{\varphi}, F_{\varphi}\right)$ for $\varphi=\varphi_{(f, F)}$.

Proof. If we replace the category fDiU used in the proof of [18, Theorem 4.8] with ifDiU, the required is clear.

Note that the categories $\mathbf{d f P D i U}, \mathbf{d f N p D i U}$ and $\mathbf{d f A p D i U}$ are equivalent since $\mathbf{d f P D i U}$ is an isomorphismclosed and full subcategory of the subcategories $\mathbf{d f N p D i U}$, dfApDiU of dfDiU consisting of nearly plain textures and almost plain textures, respectively. Therefore the category ifPDiU is equivalent to $\mathbf{d f N p D i U}$ and dfApDiU by Theorem 2.14.

Obviously, an ifDiU-isomorphism is characterized as an ifTex-isomorphism (by Proposition 1.1) which together with its inverse, is uniformly bicontinuous. Also, since the notion textural isomorphism introduced 
in [4] characterizes an fTex-isomorphism as showed in [5]. Thus, a textural isomorphism gives an ifTexisomorphism. Finally, an fDiU-isomorphism which is a textural isomorphism such that it and its inverse are uniformly continuous gives an ifDiU-isomorphism. But an ifDiU-isomorphism may not be an fDiUisomorphism.

Now let us construct the quotient category (see [13]) ifNpDiU/ $\sim_{p}$ of the di-uniform nearly plain texture spaces by using the congruence relation $\sim_{p}$ defined as $\left.\varphi \sim_{p} \psi \Longleftrightarrow \varphi\right|_{s_{p}}=\left.\psi\right|_{s_{p}}, \varphi, \psi \in \mathrm{BA}(S)$, in [24]. Therefore, we have an isomorphism similar to that given in Theorem 2.14:

Theorem 2.15. The subcategory $\mathbf{d f N p D i U}$ of $\mathbf{d f D i U}$ whose objects are di-uniform nearly plain texture spaces and morphisms are bicontinuous difunctions is isomorphic to the quotient category if $\mathrm{NpDiU} / \sim_{p}$.

Proof. First of all, we define the subconstruct ifNpTex of ifTex whose objects are nearly plain textures, morphisms are $\omega$-preserving point functions and note that the condition $\left(f_{\varphi}, F_{\varphi}\right)=\left.\left(f_{\psi}, F_{\psi}\right) \Longleftrightarrow \varphi\right|_{s_{p}}=\left.\psi\right|_{s_{p}}$ given as (2.7) in [24] by virtue of the proof of [24, Theorem 2.10] for any $\omega$-preserving point functions $\varphi$ and $\psi$. Thus, if we consider the quotient category if $\mathrm{NpDiU} / \sim_{p}$ defined above, constructed by using the equivalence relation $\sim_{p}$, a trivial functor $\Re_{u}$ from the category dfNpDiU to ifNpDiU/ $\sim_{p}$ may be established. Indeed, the functor $\Re_{u}$ is defined as similar to the functor $\Re$ between $\operatorname{dfNpTex}$ and ifNpTex/ $/ \sim_{p}$, constructed by considerations given in the last two paragraphs of [24, Page 178]. Clearly, the main difference between these functors is merely related to objects of the categories. Hence, $\Re_{u}$ is an isomorphism between $\mathbf{d f N p D i U}$ and ifNpDiU $/ \sim_{p}$ as well as $\Re$.

\section{Relations Between the Category of Quasi-Uniform Spaces and the Category ifDiU}

In this section we look at the link between di-uniformities and quasi-uniformities in a categorical setting insofar as completeness is concerned. Our point of departure will be the isomorphism mentioned below, between the category $\mathbf{Q U}_{0}$, consisting of separated quasi-uniform spaces and quasi-uniformly continuous functions and the subcategory ifPDiU $\mathbf{U}_{0}$ of ifDiU, consisting of separated di-uniform plain texture spaces and $\omega$-preserving, uniformly bicontinuous point functions.

Recall that separated di-uniformity is essentially the di-uniformity which generates a $T_{0}$ uniform ditopology. Also, the term separated quasi-uniformity means that the quasi-uniformity which generates a weakly pairwise $T_{0}$ bitopological space. Note that the separated quasi-uniformity need not correspond to a separated di-uniformity on the discrete texture $(X, \mathcal{P}(X))$. Therefore we replace $(X, \mathcal{P}(X))$ by $\left(X, \mathcal{K}_{u v}\right)$, the texture introduced in [25] such that $\mathcal{K}_{u v}$ is the smallest plain texturing of $X$ containing $u \cup v^{c}$ for a weakly pairwise $T_{0}$ bitopological space $(X, u, v)$.

On the other hand, it is mentioned in [27, Section 2] that a (separated) dual covering quasi-uniformity $Q$ has a base $\mathcal{B}$ consisting of open dual covers (see [2]) $U$ satisfying $A U B \Longrightarrow A \cap B \neq \emptyset$ for each $U \in \mathcal{B}$ and if take an open dual cover as $U=\left\{\left(A_{j}, B_{j}\right) \mid j \in J\right\}$ of $(X, u, v)$ then we have a dicover $u^{*}(U)=\left\{\left(A_{j}, X \backslash B_{j}\right) \mid j \in J\right\}$ of the plain texture $\left(X, \mathcal{K}_{u v}\right)$. Consequently, a (separated) dicovering uniformity, that is di-uniformity on $\left(X, \mathcal{K}_{u v}\right)$ is defined by the equality:

$$
u^{*}(\mathcal{Q})=\left\{\mathcal{C} \mid \mathcal{C} \text { is a dicover of }\left(X, \mathcal{K}_{u v}\right) \text { and there exists } U \in \mathcal{B} \text { with } u^{*}(U)<\mathcal{C}\right\} .
$$

On the contrary, for an open, coclosed dicover $U=\left\{\left(A_{j}, B_{j}\right) \mid j \in J\right\}$ of the space $\left(X, \mathcal{K}_{u v}, u, v^{c}\right)$, we obtain an open dual cover ${ }^{*} u(U)$ of $(X, u, v)$ by setting ${ }^{*} u(U)=\left\{\left(A_{j}, X \backslash B_{j}\right) \mid j \in J\right\}$. Hence, for a (separated) dicovering uniformity $\mathcal{U}$ on $\left(X, \mathcal{K}_{u v}\right)$ we have a (separated) dual covering quasi-uniformity

$$
{ }^{*} u(\mathcal{U})=\left\{\gamma \mid \gamma \text { is a dual cover of } X \text { and there exists } U \in \mathcal{U} \text { with }{ }^{*} u(U)<\gamma\right\}
$$

on $(X, u, v)$, generated by the dual covers ${ }^{*} u(U)$ for an open, coclosed and anchored dicover $U \in \mathcal{U}$. Finally, it is shown easily that the mapping $u^{*}$ is a bijection with its inverse ${ }^{*} u$, between the (separated) dual covering quasi-uniformities on $X$ and the (separated) dicovering uniformities on $\left(X, \mathcal{K}_{u v}\right)$.

As a result of these facts, we have the following isomorphism. For the proof and the other useful details see [27, Theorem 2.15] in a wider context. 
Theorem 3.1. The functor $\mathfrak{H}_{u}: \mathbf{Q U}_{0} \longrightarrow$ ifPDiU $_{0}$

$$
\mathfrak{S}_{u}\left((X, Q) \stackrel{\varphi}{\rightarrow}\left(Y, Q^{\prime}\right)\right)=\left(X, \mathcal{K}_{\tau_{Q} \tau_{Q^{-1}}}, u^{*}(Q)\right) \stackrel{\varphi}{\rightarrow}\left(Y, \mathcal{K}_{\tau_{Q^{\prime}} \tau_{Q^{\prime}-1}}, u^{*}\left(Q^{\prime}\right)\right)
$$

is faithfull, full and bijective on objects, that is, $\mathfrak{H}_{u}$ is an isomorphism.

Therefore, the concrete categories $\mathbf{Q U}_{\mathbf{0}}$ and $\mathbf{i f P D i \mathbf { U } _ { 0 }}$ are concretely isomorphic.

On the way, we will give some results by using the functor $\mathfrak{H}_{u}$. Firstly, recall the following commutative diagram mentioned in [27]:

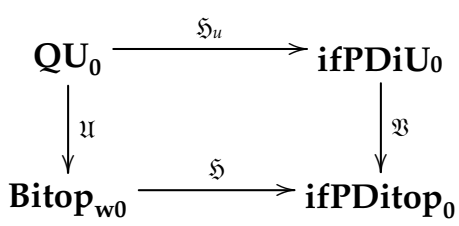

For all categories and functors showed in the diagram, see [27, Theorem 2.15].

Now let us turn our attention to the completeness for quasi-uniform spaces and di-uniform texture spaces.

First of all, we have the notions of bicompleteness in the sense of Fletcher [10] and s-completeness in the sense of Brümmer [8]. These notions were used for the completeness of quasi-uniform spaces as " $a$ quasi-uniform space $(X, Q)$ is bicomplete (s-complete) if and only if the corresponding uniform space $\left(X, Q \vee Q^{-1}\right)$ is complete". Here $Q^{-1}$ denotes the conjugate of $Q$, in other words, it is defined as $\left\{q^{-1} \mid q \in Q\right\}$ where $q^{-1}=\{(y, x) \mid(x, y) \in q\}$. In addition, $Q \vee Q^{-1}$ denotes the quasi-uniformity whose base is $\left\{q \cap q^{-1} \mid q \in Q\right\}$ and which is also coarsest uniformity contains $Q$ and $Q^{-1}$ for the quasi-uniformity $Q$.

Note that the term pairwise complete given in [9] is used instead of s-complete in the same sense.

On the other hand, we recall another type of completeness for the quasi-uniform spaces, under the name Q-completeness (see [2]) as follows:

Definition 3.2. A quasi-uniform space $(X, Q)$ is called Q-complete if every 1-regular Q-Cauchy bifilter is convergent in the bitopological space $\left(X, \tau_{\Omega}, \tau_{Q^{-1}}\right)$.

Particularly, this notion coincides with the notion of B-completeness (double completeness) in the sense of [14]. Because the notions of Cauchy filter pair given in [15] and Cauchy bifilter given in [2] are identical for a diagonal quasi-uniformity. Indeed, note that for a dual-cover $d \in Q$, a diagonal is defined as $U=$ $\bigcup_{(F, G) \in d}(G \times F)$ in $[2]$.

For the remainder of paper, we will use the term "Q-complete" given in Definition 3.2, for the completeness of quasi-uniform space $(X, 2)$.

Also, an appropriate notion for completeness of di-uniform texture spaces is defined under the name dicompleteness and also obtained some useful results in [19] as mentioned in Section 1, as well.

Now, we require the following lemma given as [27, Proposition 2.16].

Lemma 3.3. The space $(X, Q) \in \mathrm{Ob} \mathrm{QU}_{\mathbf{0}}$ is Q-complete if and only if the space $\mathfrak{H}_{u}(X, Q)=\left(X, \mathcal{K}_{\tau_{Q} \tau_{Q^{-1}}}, u^{*}(Q)\right) \in$ $\mathrm{Ob}$ ifPDiU $\mathrm{U}_{0}$ is dicomplete.

Note that, in this work we will say that $\left(Y, Q^{\prime}\right) \in \mathrm{Ob} \mathbf{Q U}_{\mathbf{0}}$ is Q-completion of $(X, Q) \in \mathrm{Ob} \mathbf{Q U}_{\mathbf{0}}$ if the space $(X, Q)$ is quasi-uniform isomorphic to a jointly dense subspace (that is, dense with respect to the joint topology $\left.\tau_{Q^{\prime}} \vee \tau_{Q^{\prime-1}}\right)$ of the $Q^{\prime}$-complete space $\left(Y, Q^{\prime}\right)$.

Additionally, recall the notion of plain dicompletion from [27] for di-uniform texture spaces. Therefore, by using the definition of functor $\mathfrak{S}_{u}$ set up in Theorem 3.1 together with Lemma 3.3 and [27, Theorem 2.5, Lemmas $2.13,2.14]$, now we can state the following characterization clearly: 
Proposition 3.4. $\left(Y, Q^{\prime}\right) \in \mathrm{Ob} \mathbf{Q U}_{0}$ is a $Q$-completion of $(X, Q) \in \mathrm{Ob}_{\mathbf{Q U}}$ if and only if the space $\mathfrak{H}_{u}\left(Y, Q^{\prime}\right)=$ $\left(Y, \mathcal{K}_{\tau_{Q^{\prime}} \tau_{Q^{\prime}-1}}, u^{*}\left(Q^{\prime}\right)\right) \in \mathrm{Ob}_{\text {ifPDiU }}$ is a plain dicompletion of $\mathfrak{S}_{u}\left(Y, Q^{\prime}\right)=\left(X, \mathcal{K}_{\tau_{Q} \tau_{Q^{-1}}}, u^{*}(Q)\right) \in \mathrm{Ob}_{\text {ifPDiU }}$.

According to the above proposition, we have a next statement:

Proposition 3.5. If $(T, \mathcal{T}, \mathcal{V}) \in \mathrm{Ob}_{\text {ifPDiU }}$ is a plain dicompletion of $(S, \mathcal{S}, \mathcal{U}) \in \mathrm{Ob}_{\text {ifPDiU }}$ then the corresponding space $(T, * u(\mathcal{V})) \in \mathrm{Ob} \mathbf{Q U}_{0}$ is a ${ }^{*} u(\mathcal{U})$-completion of $\left(S,{ }^{*} u(\mathcal{U})\right) \in \mathrm{Ob}_{\mathbf{Q U}}$.

Proof. It is clear from Proposition 3.4 and [25, Corollary 3.8].

Recall that for a separated quasi-uniform space $(X, Q)$, we have a weakly pairwise $T_{0}$ bitopological space $\left(X, \tau_{Q}, \tau_{Q^{-1}}\right)$ and a separated di-uniform plain texture space $\left(X, \mathcal{K}_{\tau_{\Omega} \tau_{Q^{-1}}}, u^{*}(Q)\right)$ as well. Note here that $\tau_{u^{*}(Q)}=$ $\tau_{Q}, \kappa_{u^{*}(\mathcal{Q})}=\left(\tau_{Q^{-1}}\right)^{c}$. In addition, if we consider the T-lattice $P(X)\left(P^{*}(X)\right)$ of pairwise continuous, real-valued (bounded) functions defined on the space $\left(X, \tau_{Q}, \tau_{Q^{-1}}\right)$ then the equality $P(X)=\mathrm{BA}(X)\left(P^{*}(X)=\mathrm{BA}^{*}(X)\right)$ is clear. Hence in particular, if we take $Q$ as the initial quasi-uniformity $Q_{P(X)}\left(Q_{P^{*}(X)}\right)$ generated by the family $P(X) \quad\left(P^{*}(X)\right)$ then the corresponding di-uniformity $u^{*}\left(Q_{P(X)}\right)\left(u^{*}\left(Q_{P^{*}(X)}\right)\right)$ on the texture $\left(X, \mathcal{K}_{\tau_{Q} \tau_{Q_{-1}}}\right)$ is the same with initial di-uniformity $\mathcal{U}_{\mathrm{BA}(X)}\left(\mathcal{U}_{\mathrm{BA}^{*}(X)}\right)$ generated by the $T$-lattice $\mathrm{BA}(X)\left(\mathrm{BA}^{*}(X)\right)$. Conversely, it is straightforward to verify that if $\mathcal{U}$ is initial di-uniformity on a plain texture $(S, \mathcal{S})$ then ${ }^{*} u(\mathcal{U})$ is initial quasi-uniformity on the set $S$ such that $\tau_{u}=\tau_{*} u(u)$ and $\kappa_{u}=\left(\tau_{*} u(\mathcal{u})^{-1}\right)^{c}$.

It is easy to prove that the quasi-uniform space $(X, Q)$ is $Q$-complete then the corresponding uniform space $\left(X, Q \vee Q^{-1}\right)$ is complete. Therefore, if $\left(Y, Q^{\prime}\right)$ is a $Q$-completion of the space $(X, Q)$ then the uniform space $\left(Y, Q^{\prime} \vee Q^{\prime-1}\right)$ is the uniform completion of the uniform space $\left(X, Q \vee Q^{-1}\right)$ since a quasi-uniformly continuous mapping between quasi-uniform spaces is uniformly continuous between corresponding uniform spaces.

On the contrary, a quasi-uniform space $(X, Q)$ may not be $Q$-complete even though its corresponding uniform space $\left(X, Q \vee Q^{-1}\right)$ is complete, that is $(X, Q)$ is bicomplete. In fact, H.P.A. Künzi gave a counterexample (see Example 5.5) for it, as Sorgenfrey quasi-uniform space and he mentioned that Sorgenfrey quasi-uniform space is not $B$-complete (that is, not $Q$-complete in the sense of Definition 3.2) even though its corresponding uniform space is discrete and thus (uniform) complete, that is, Sorgenfrey quasi-uniform space is bicomplete.

Now let us consider the above facts in a textural point of view:

Clearly, di-uniformities are the textural analogue of quasi-uniformities because of Theorem 3.1. But it is not trivial that whether there is an analogous link between uniform spaces and di-uniform plain texture spaces.

Even so, note that this work is related with the connections between the completeness of (quasi-)uniform spaces and the completeness of di-uniform texture spaces. Therefore as first step in this section, we have mentioned that the relationships between quasi-uniformities and di-uniformities insofar as completeness is interested.

Following this, in a similar manner, we will investigate the link between the completeness of uniform spaces and a special type of completeness of the di-uniform texture spaces. As a principle way, our point of departure will be the statement complemented di-uniformities, that is the di-uniformities endowed with a complementation are the textural analogue of uniformities, proved in [17]. This shows the pervasive role played by complementation.

Accordingly, in the next section we will especially deal with the notions complementation, complemented textures [5] and complemented di-uniformities [17].

\section{The Category ifCDiU}

In this section, after recalling the definitions of complementation, complemented di-uniform texture space and the other useful notions related with complementation, we will introduce a category of complemented di-uniform discrete texture spaces and $\omega$-preserving, uniformly bicontinuous point functions (not necessarily complemented point function defined in [5, Page 194]) and study on its some properties. We begin by defining the notion of complementation (see [5]) as follows: 
Definition 4.1. First of all, note that the texturing $\mathcal{S}$ on a set $S$ need not be closed under the set-complementation in general, but a mapping $\sigma: \mathcal{S} \rightarrow \mathcal{S}$ satisfying the conditions

i) $\sigma(\sigma(A))=A$

ii) $A \subseteq B \Longrightarrow \sigma(B) \subseteq \sigma(A), \forall A, B \in \mathcal{S}$

can be defined on the texturing $\mathcal{S}$. In this case $\sigma$ is called complementation on $(S, \mathcal{S})$, and the triple $(S, \mathcal{S}, \sigma)$ a complemented texture.

In the present paper, we will use the notation ifCTex for the category of complemented textures and $\omega$-preserving point functions.

Additionally, a ditopological texture space $(S, \mathcal{S}, \tau, \kappa)$ with the complementation $\sigma$ on $(S, \mathcal{S})$ is called complemented ditopological texture space and the ditopology $(\tau, \kappa)$ complemented ditopology if the condition $\kappa=\sigma(\tau)$ is satisfied.

Examples 4.2. (1) For a subset $Y \subseteq X$, set difference $\pi(Y)=X \backslash Y$ is a complementation on the discrete texture $(X, \mathcal{P}(X))$ given in Examples $1.2(1)$. It is the existence of this complementation that is responsible for many of the special properties of this texture, particularly in relation to symmetry. In addition, for any topology $\mathcal{T}$ on $X,\left(\mathcal{T}, \mathcal{T}^{c}\right)$ is a complemented ditopology on $(X, \mathcal{P}(X), \pi) \in$ Ob ifCTex, where $\mathcal{T}^{c}=\pi(\tau)=\{X \backslash G \mid G \in \mathcal{T}\}$.

(2) A complementation on the real texture $(\mathbb{R}, \mathcal{R})$ given in Examples $1.2(2)$ is defined by setting

$$
\gamma((-\infty, r))=(-\infty,-r] \text { and } \gamma((-\infty, r])=(-\infty,-r) \text { for all } r \in \mathbb{R} \text {. }
$$

Moreover, the natural ditopology $\left(\tau_{\mathbb{R}}, \kappa_{\mathbb{R}}\right)$ is complemented on $(\mathbb{R}, \mathcal{R}, \gamma) \in$ Ob ifCTex.

(3) A complementation on the unit interval texture (II, J) given in Examples 1.2 (3), is defined by setting

$$
\iota([0, r])=[0,1-r) \text { and } \iota([0, r))=[0,1-r] \text { for all } r \in \mathbb{I},
$$

and $\tau_{\mathbb{I}}=\{[0, r) \mid 0 \leq r \leq 1\} \cup\{\mathbb{I}\}, \kappa_{\mathbb{I}}=\{[0, r] \mid 0 \leq r \leq 1\} \cup\{\emptyset\}$ defines the complemented ditopology $\left(\tau_{\mathbb{I}}, \kappa_{\mathbb{I}}\right)$ on $(\mathbb{I}, \mathcal{J}, \iota) \in \mathrm{Ob}$ ifCTex.

Therefore, the above examples shows that indeed many of the naturally occurring textures can be equipped with a complementation.

Now we can recall from [17] the effect of a complementation on a di-uniformity as follows:

Definition 4.3. For a given direlational uniformity $\mathcal{U}$ on $(S, \mathcal{S}, \sigma) \in$ Ob ifCTex, the direlational uniformity $\sigma(\mathcal{U})=$ $\mathcal{U}^{\prime}=\left\{(d, D)^{\prime} \mid(d, D) \in \mathcal{U}\right\}$ is called complement of $\mathcal{U}$ and if the equality $\mathcal{U}^{\prime}=\mathcal{U}$ is satisfied then $\mathcal{U}$ is said to be complemented direlational uniformity. Here the notation $\sigma(d, D)=(d, D)^{\prime}$ defined in [17] denotes the complement of direlation $(d, D) \in \mathcal{U}$.

We know from [17], for each direlational uniformity $\mathcal{U}$, the uniform ditopology of the direlational uniformity $\mathfrak{U}^{\prime}=\sigma(\mathcal{U})$ is defined by the equality $\left(\tau_{\mathcal{U}^{\prime}}, \kappa_{\mathcal{U}^{\prime}}\right)=\left(\sigma\left(\kappa_{\mathcal{U}}\right), \sigma\left(\tau_{\mathcal{U}}\right)\right)$. In addition, for a direlational uniformity $\mathcal{U}$ on $(S, \mathcal{S}, \sigma) \in \mathrm{Ob}$ ifCTex, a complemented direlational uniformity with respect to $\sigma$ is defined on $S$ with the equality

$$
\mathcal{U} \vee \sigma(\mathcal{U})=\{(d, D) \mid \exists(e, E) \in \mathcal{U} \text { with }(e, E) \sqcap \sigma(e, E) \sqsubseteq(d, D)\} .
$$

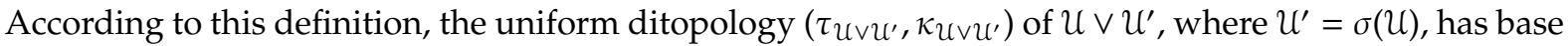
$\left\{G \cap \sigma(K) \mid G \in \tau_{\mathcal{U}}, K \in \mathcal{K}_{\mathcal{U}}\right\}$ and cobase $\left\{K \cup \sigma(G) \mid K \in \mathcal{K}_{\mathcal{U}}, G \in \tau_{\mathcal{U}}\right\}$. Hence it is clear that the topologies

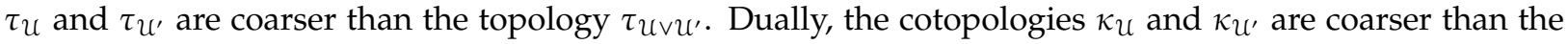
cotopology кuvน'.

By combining these considerations with the definition of Cauchy difilter given in the first section we arrive at the following: 
Lemma 4.4. Let $\mathcal{F} \times \mathcal{G}$ be a difilter and $\mathcal{U}$ a direlational uniformity on $(\mathcal{S}, \mathcal{S}, \sigma) \in \mathrm{Ob}$ ifCTex. If $\mathcal{F} \times \mathcal{G}$ is $\mathcal{U} \vee \sigma(\mathcal{U})$ Cauchy then $\mathcal{F} \times \mathcal{G}$ is U-Cauchy.

Proof. Obvious from the fact that if $(d, D) \in \mathcal{U}$ then $(d, D) \in \mathcal{U} \vee \sigma(\mathcal{U})$.

On the other hand, recall the complement of dicover $\mathcal{C}=\left\{\left(A_{j}, B_{j}\right) \mid j \in J\right\}$ on the complemented texture $(S, \mathcal{S}, \sigma)$ is defined as $\mathfrak{C}^{\prime}=\sigma(\mathcal{C})=\left\{\left(\sigma\left(B_{j}\right), \sigma\left(A_{j}\right)\right) \mid j \in J\right\}$ and it is easy to verify that $\mathcal{C}^{\prime}$ is also a dicover on $(S, \mathcal{S}, \sigma)$ and similar to the procedures given in Definition 4.3, we may now describe what we should mean by the complement of a dicovering uniformity:

Definition 4.5. Let $\mathcal{V}$ be a dicovering uniformity on $(S, \mathcal{S}, \sigma) \in \mathrm{Ob}$ ifCTex. Then the complement of $\mathcal{V}$ is the dicovering uniformity $\sigma(\mathcal{V})=\mathcal{V}^{\prime}$ with the base $\left\{\left(\mathcal{C}^{\prime}\right)^{\Delta} \mid \mathcal{C} \in \mathcal{V}\right\}$ and $\mathcal{V}$ is called complemented dicovering uniformity if $\mathcal{V}^{\prime}=\mathcal{V}$. (Here, see [16] for the notation $\left.\left(\mathrm{C}^{\prime}\right)^{\Delta}\right)$

Note 4.6. From now on, we will use the term complemented di-uniformity to refer to complemented dicovering uniformity and complemented direlational uniformity.

Hence the next definition may be stated:

Definition 4.7. The texture $(S, \mathcal{S}, \sigma) \in \mathrm{Ob}$ ifCTex with a di-uniformity $U$ complemented with respect to the complementation $\sigma$ is called complemented di-uniform texture space and denoted by the notation $(\mathcal{S}, \mathcal{S}, \sigma, \mathcal{U})$.

By regarding the above facts, we will denote by ifCDiU the category of complemented di-uniform texture spaces and $\omega$-preserving, uniformly bicontinuous point functions. It is trivial that ifCDiU is concrete category over ifCTex.

Note that the category ifCTex has products since the product of complemented textures is complemented and the projection map is complemented as mentioned in [5]. Therefore, the following result is now natural by using Corollary 2.11:

Corollary 4.8. The category ifCDiU has products.

Additionally, note that for the space $(S, \mathcal{S}, \sigma, \mathcal{U}) \in \mathrm{Ob}$ ifCDiU, the ditopology $\left(\tau_{\mho}, \kappa_{\varkappa}\right)$ generated by the complemented di-uniformity $\mathcal{U}$ is a complemented ditopology, that is $\kappa_{\mathcal{U}}=\sigma\left(\tau_{\mathcal{U}}\right)$. Thus, it may be seen easily that there is a concrete functor between ifCDiU and the category ifCDitop whose objects are complemented ditopological texture spaces.

Also, in order to use in the remainder of this paper, we consider the full subcategory ifDDiU of ifDiU whose objects are di-uniform discrete texture spaces and morphisms are $\omega$-preserving, uniformly bicontinuous point functions.

Therefore the following result arises:

Theorem 4.9. ifDDiU is a concretely coreflective subcategory of ifPDiU.

Proof. It is similar to the proof of [18, Theorem 3.7] because of the explicit isomorphisms between the categories ifDDiU and dfDDiU, also the categories ifPDiU and dfPDiU.

In addition we may give:

Proposition 4.10. Let $U$ be a di-uniformity on the complemented ditopological discrete texture space $\left(S, \mathcal{P}(S), \pi, \tau, \tau^{c}\right)$ such that $\tau=\tau_{\mathfrak{U}}, \tau^{c}=\kappa_{\mathfrak{U}}=\pi(\tau)$. If $(S, \mathcal{P}(S), \mathcal{U}) \in$ Ob ifDDiU is dicomplete then $\left(S, \mathcal{P}(S), \mathcal{U} \vee \mathfrak{U}^{\prime}\right) \in$ Ob ifDDiU is dicomplete, where $\mathfrak{U}^{\prime}=\pi(\mathfrak{U})$.

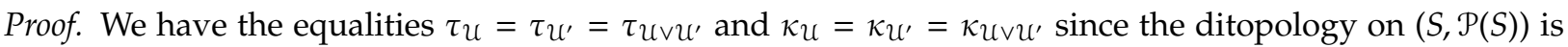
complemented. Now let the difilter $\mathcal{F} \times \mathcal{G}$ be $\mathcal{U} \vee \mathfrak{U}^{\prime}$-Cauchy where $\mathfrak{U}^{\prime}=\pi(\mathcal{U})$. Thus by Lemma $4.4, \mathcal{F} \times \mathcal{G}$ is a $\mathcal{U}$-Cauchy difilter. Also, since $(S, \mathcal{P}(S), \mathcal{U}) \in \mathrm{Ob}$ ifDDiU is dicomplete, $\mathcal{F} \times \mathcal{G}$ is diconvergent with respect to the ditopology $\left(\tau_{u}, \kappa_{\mho}\right)$. In this case, $\mathcal{F} \times \mathcal{G}$ is diconvergent with respect to the ditopology $\left(\tau_{u v u^{\prime}}, \kappa_{u v u^{\prime}}\right)$

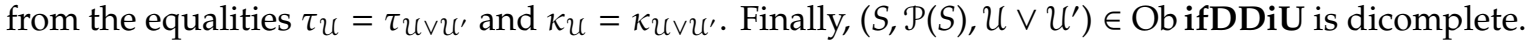


Now we will investigate the effect of a complementation on a difilter. For this, we introduce with the notions $\sigma$-complement of a filter $\mathcal{F}$ and $\sigma$-complement of a cofilter $\mathcal{G}$ on the complemented texture $(S, \mathcal{S}, \sigma)$ as $\sigma(\mathcal{F})=\{\sigma(F) \mid F \in \mathcal{F}\}$ and $\sigma(\mathcal{G})=\{\sigma(G) \mid G \in \mathcal{G}\}$, respectively. Hence, it is clear that $\sigma(\mathcal{F})$ is a cofilter and $\sigma(\mathcal{G})$ is a filter on $(S, \mathcal{S}, \sigma)$.

In the light of these considerations we may define $\sigma$-complement of the difilter $\mathcal{F} \times \mathcal{G}$ on a complemented texture endowed with the complementation $\sigma$ by the equality

$$
\sigma(\mathcal{F} \times \mathcal{G})=\sigma(\mathcal{G}) \times \sigma(\mathcal{F}) .
$$

According to this, the following proposition is straightforward.

Proposition 4.11. Let $\mathcal{F} \times \mathcal{G}$ be a difilter and $\mathcal{U}$ a di-uniformity on $(S, \mathcal{S}, \sigma) \in \mathrm{Ob}$ ifCTex. In this case, $\mathcal{F} \times \mathcal{G}$ is $\mathcal{U}$-Cauchy if and only if $\sigma(\mathcal{F} \times \mathcal{G})$ is $\sigma(\mathcal{U})$-Cauchy.

Practically let us state the next definition:

Definition 4.12. Let $(S, \mathcal{S}, \sigma) \in \mathrm{Ob}$ ifCTex. Any difilter $\mathcal{F} \times \mathcal{G}$ on $(S, \mathcal{S})$ is called $\sigma$-complemented difilter if $\sigma(\mathcal{F} \times \mathcal{G})=$ $\mathcal{F} \times \mathcal{G}$.

With this definition, it is easy to verify that if $\mathcal{F} \times \mathcal{G}$ is a regular $\sigma$-complemented difilter on $(S, \mathcal{S}, \sigma)$ then $\sigma(\mathcal{F} \times \mathcal{G})$ is a regular $\sigma$-complemented difilter on $(S, \mathcal{S}, \sigma)$.

Moreover, if the difilter $\mathcal{F} \times \mathcal{G}$ on the discrete space $(S, \mathcal{P}(S), \pi) \in \mathrm{Ob}$ ifCTex is $\pi$-complemented then it is regular difilter, clearly.

Now we can establish some examples:

Example 4.13. Let $L=(0,1]$ and $\mathcal{L}=\{(0, r] \mid 0 \leq r \leq 1\}$, where $(0,0]$ is interpreted as $\emptyset$. Then $(L, \mathcal{L})$ is a texture with $P_{r}=Q_{r}=(0, r]$ for all $r \in L$ and it is complemented texture with the complementation $\lambda$, defined as $\lambda(0, r]=(0,1-r]$ on $(L, \mathcal{L})$. Now if we define $\mathcal{F}=\{(0, r] \mid 1 / 2<r \leq 1\}$ and $\mathcal{G}=\{(0, r] \mid 0 \leq r<1 / 2\}$, it is clear that $\mathcal{F} \times \mathcal{G}$ is a regular $\lambda$-complemented difilter.

Example 4.14. On the complemented ditopological discrete texture space $\left(S, \mathcal{P}(S), \pi, \tau, \tau^{c}\right)$ where $\tau^{c}=\pi(\tau)$, the difilter $\eta(s) \times \mu(s)$ defined in [19] is a regular $\pi$-complemented difilter.

By virtue of Definition 4.12, we have the following characterization which contains the converse of Lemma 4.4 on the one hand.

Lemma 4.15. Let $\mathcal{F} \times \mathcal{G}$ be a $\sigma$-complemented difilter and $\mathcal{U}$ a di-uniformity on $(S, \mathcal{S}, \sigma) \in \mathrm{Ob}$ ifCTex. Thus, $\mathcal{F} \times \mathcal{G}$ is $\mathcal{U}$-Cauchy if and only if $\mathcal{F} \times \mathcal{G}$ is $\mathcal{U} \vee \mathcal{U}^{\prime}$-Cauchy, where $\mathfrak{U}^{\prime}=\sigma(\mathcal{U})$.

Proof. Necessity. If take $(d, D) \in \mathcal{U} \vee \mathcal{U}^{\prime}$ then there is a direlation $(e, E) \in \mathcal{U}$ such that $(e, E) \sqcap(e, E)^{\prime} \sqsubseteq(d, D)$ and there exists $s \in S$ such that $e[s] \in \mathcal{F}, E[s] \in \mathcal{G}$ since $\mathcal{F} \times \mathcal{G}$ is $\mathcal{U}$-Cauchy, thus $e^{\prime}[s] \in \sigma(\mathcal{F}), E^{\prime}[s] \in \sigma(\mathcal{G})$. On the other hand, in view of the fact that $\sigma(\mathcal{F})=\mathcal{G}$ and $\sigma(\mathcal{G})=\mathcal{F}$, clearly $E^{\prime}[s] \in \mathcal{F}$ and $e^{\prime}[s] \in \mathcal{G}$. Consequently $d[s] \in \mathcal{F}$ and $D[s] \in \mathcal{G}$, that is $\mathcal{F} \times \mathcal{G}$ is $\mathcal{U} \vee \mathfrak{U}^{\prime}$-Cauchy.

Sufficiency is clear from Lemma 4.4 .

Now, by recalling from [19] the definition of diconvergency of a difilter, a special type of completeness in the category ifCDiU is introduced as follows:

Definition 4.16. Let $(S, S, \sigma, \mathcal{U}) \in \mathrm{Ob}$ ifCDiU. If every regular $\sigma$-complemented $\mathcal{U}$-Cauchy difilter is diconvergent in $S$ then the space $(S, \mathcal{S}, \sigma, \mathcal{U})$ is called $\sigma$-complete.

Because of this definition, the following proposition is obvious:

Proposition 4.17. Let $(S, \mathcal{S}, \sigma, \mathcal{U}) \in \mathrm{Ob}$ ifCDiU. If the space $(S, \mathcal{S}, \mathcal{U}) \in \mathrm{Ob}$ ifDiU is dicomplete then $(S, \mathcal{S}, \sigma, \mathcal{U})$ is $\sigma$-complete. 
For the converse of Proposition 4.17 we will state a counterexample in the final section. Now, with the help of Definition 4.16 we may describe a next notion as follows:

Definition 4.18. The space $(S, \mathcal{S}, \tau, \kappa) \in \mathrm{Ob}$ ifDitop with a complementation $\sigma$ on the texture $(S, \mathcal{S})$ is called ditopologically complete if there exists a di-uniformity $u$ such that $\tau=\tau_{\mathcal{U}}, \kappa=\kappa_{\mathfrak{U}}$ and the complemented di-uniform texture space $\left(S, \mathcal{S}, \sigma, \mathcal{U} \vee \mathcal{U}^{\prime}\right) \in \mathrm{Ob}$ ifCDiU is $\sigma$-complete, where $\mathcal{U}^{\prime}=\sigma(\mathfrak{U})$.

This definition leads to the following statement:

Proposition 4.19. Let $(S, \mathcal{S}, \sigma, \mathcal{U}) \in \mathrm{Ob}$ ifCDiU. If $(S, \mathcal{S}, \mathcal{U})$ is dicomplete then the corresponding space $\left(S, \mathcal{S}, \tau, \tau_{\mathcal{U}}, \mathcal{\varkappa}\right) \in$ $\mathrm{Ob}$ ifDitop with the complementation $\sigma$ is ditopologically complete.

Proof. Straightforward from Proposition 4.17 and the equality $U=\sigma(\mathcal{U})=U \vee \sigma(U)$.

Therefore, we will present an example for the notion introduced in Definition 4.18.

Example 4.20. If we consider the real ditopological plain texture space $\left(\mathbb{R}, \mathcal{R}, \tau_{\mathbb{R}}, \kappa_{\mathbb{R}}\right)$ given in Examples $1.2(2)$, as it is shown in $[27$, Lemma 3.1] the texture $(\mathbb{R}, \mathcal{R})$ has a dicomplete dicovering uniformity whose uniform ditopology coincides with the real ditopology $\left(\tau_{\mathbb{R}}, \kappa_{\mathbb{R}}\right)$. This di-uniformity has the base $\left\{\mu_{\epsilon} \mid \epsilon>0\right\}$, where $\mu_{\epsilon}=\left\{\left(Q_{x+\epsilon}, P_{x-\epsilon}\right) \mid\right.$ $x \in \mathbb{R}\}$ and is denoted by $v_{\mathbb{R}}$ under the name standard di-uniformity on $(\mathbb{R}, \mathcal{R})$.

Furthermore, if the mapping $\pi$ on $(\mathbb{R}, \mathcal{R})$ is considered as a complementation, the di-uniform space $\left(\mathbb{R}, \mathcal{R}, \pi, v_{\mathbb{R}}\right) \in$ $\mathrm{Ob}$ ifCDiU is $\pi$-complete by Proposition 4.17 since $\left(\mathbb{R}, \mathcal{R}, v_{\mathbb{R}}\right)$ is dicomplete. Hence, $\left(\mathbb{R}, \mathcal{R}, \tau_{\mathbb{R}}, \kappa_{\mathbb{R}}\right) \in$ Ob ifDitop is ditopologically complete with the complementation $\pi$ by Proposition 4.19.

Now we define another concept required for the remainder of this paper.

Definition 4.21. The space $(S, S, \tau, \kappa) \in \mathrm{Ob}$ ifDitop is called difilter complete if there exists a di-uniformity $U$ such that $\tau=\tau_{u}, \kappa=\kappa_{u}$ and $(S, \mathcal{S}, \mathcal{U}) \in \mathrm{Ob}$ ifDiU is dicomplete.

Therefore the following characterization is clear.

The space $(S, S, \mathcal{U}) \in \mathrm{Ob}$ ifDiU is dicomplete if and only if the corresponding space $\left(S, S, \tau_{\mathcal{U}}, \kappa_{\mathcal{U}}\right) \in \mathrm{Ob}$ ifDitop is difilter complete.

Particularly, by combining the above considerations we arrive at the following result:

Corollary 4.22. Let $\left(S, \mathcal{P}(S), \tau, \tau^{c}\right) \in \mathrm{Ob}$ ifDitop with the complementation $\pi$. If the space $\left(S, \mathcal{P}(S), \tau, \tau^{c}\right)$ is difilter complete then it is ditopologically complete.

Proof. Obvious from Propositions 4.10, 4.19.

\section{Connections Between the Category of Uniform Spaces and the Category ifCDiU}

In this final section, we begin by investigating how the relationship between uniformities and complemented di-uniformities appears insofar as completeness is concerned. Additionally, some requirements between the various kinds of completeness of a di-uniform texture space and real dicompactness of the corresponding ditopological texture space generated by that di-uniformity are presented as a diagram.

Now, first of all we define the full subcategory if $\mathbf{D}_{\pi} \mathbf{D i U}$ of ifCDiU, such that its objects are $\pi$ complemented di-uniform discrete texture spaces and morphisms are $\omega$-preserving, uniformly bicontinuous point functions.

For the space $(S, \mathcal{P}(S), \mathcal{U}) \in \mathrm{Ob}$ ifDDiU, a dual-covering quasi-uniformity in the sense of [2] is defined on $S$ with the equality:

$$
\underline{u}(\mathcal{U})=\{\gamma \mid \gamma \text { is a dual cover of } X \text { and there exists } \mathcal{C} \in \mathcal{U} \text { with } \underline{u}(\mathcal{C})<\gamma\}
$$


where $\underline{u}(\mathcal{C})=\left\{\left(A_{j}, S \backslash B_{j}\right) \mid j \in J\right\}$ is a dual cover appropriate for the dicover $\mathcal{C}=\left\{\left(A_{j}, B_{j}\right) \mid j \in J\right\}$ of the discrete texture $(S, \mathcal{P}(S))$.

In particular, recall from [17] that a di-uniformity is complemented if and only if the corresponding quasi-uniformity is a uniformity. Therefore $(S, \underline{u}(\mathcal{U})) \in \mathrm{Ob}$ Uni for the space $(S, \mathcal{P}(S), \pi, \mathcal{U}) \in \mathrm{Ob}_{\text {ifD }} \mathrm{DiU}_{\text {. }}$

On the contrary, for a uniformity $\nu$ on the set $S$, we have the uniform topology $\tau_{v}$ and complemented ditopological discrete texture space $\left(S, \mathcal{P}(S), \pi, \tau_{\mathcal{V}},\left(\tau_{\mathcal{V}}\right)^{c}\right)$, also complemented di-uniformity $\bar{u}(\mathcal{V})$. (Note here that the notation $\bar{u}$ is used as $u^{*}$ in [17, Section 3])

To sum up, $(S, \mathcal{P}(S), \pi, \bar{u}(\mathcal{V})) \in \mathrm{Ob}_{\text {ifD }}$ DiU for the space $(S, \mathcal{V}) \in \mathrm{Ob}$ Uni.

Now, let us give the following isomorphism as a crucial aim:

Theorem 5.1. The functor $\mathfrak{X}_{u}: \mathrm{Uni} \longrightarrow$ ifD $\mathrm{D}_{\pi} \mathrm{DiU}$

$$
\mathfrak{x}_{u}\left((S, \mathcal{V}) \stackrel{\varphi}{\rightarrow}\left(T, \mathcal{V}^{\prime}\right)\right)=\left(S, \mathcal{P}(S), \pi_{S}, \bar{u}(\mathcal{V})\right) \stackrel{\varphi}{\rightarrow}\left(T, \mathcal{P}(T), \pi_{T}, \bar{u}\left(\mathcal{V}^{\prime}\right)\right)
$$

is faithfull, full and bijective on objects, that is, $\mathfrak{X}_{u}$ is an isomorphism.

Proof. Straightforward from the above considerations related to the mappings $\underline{u}$ and $\bar{u}$.

Hence the categories Uni and if $\mathbf{D}_{\pi} \mathbf{D i U}$ are isomorphic because of the bijective mappings $\underline{u}$ and $\bar{u}$ defined between uniformities on $S$ and $\pi$-complemented di-uniformities on $(S, \mathcal{P}(S))$. In a similar manner, the full subcategories $\mathrm{Uni}_{0}$ and $\mathrm{ifD}_{\pi} \mathrm{DiU}_{\mathbf{0}}$ of $\mathrm{Uni}_{\text {and }} \mathrm{ifD}_{\pi} \mathrm{DiU}$, respectively, whose objects are separated spaces, are isomorphic by the equality $\mathcal{K}_{\tau_{v} \tau_{v}}=\mathcal{P}(S)$ for a separated uniformity $\mathcal{V}$.

In conclusion, now we have a commutative diagram:

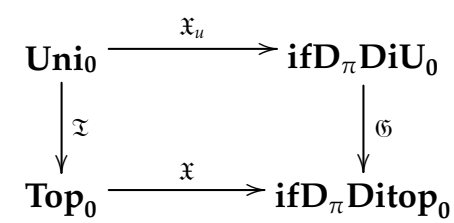

Note here that the functor $\mathfrak{X}$ is also an isomorphism and the functor $\mathfrak{X}_{u}$ is the restriction to uniform spaces of the functor $\mathfrak{S}_{u}$ given in Theorem 3.1. Here, also the notation ifD $\mathbf{D}_{\pi} \mathbf{D i t o p}_{0}$ denotes the category of $\pi$-complemented $T_{0}$ ditopological discrete texture spaces and $\omega$-preserving, bicontinuous point functions while Top $_{0}$ denotes the category of $T_{0}$ topological spaces and continuous functions. Also we may regard $\mathrm{Uni}_{0}$ as a concrete category over $\mathrm{Top}_{0}$ via the functor $\mathfrak{I}$,

$$
\mathfrak{I}\left(\left(X_{1}, \mathcal{U}_{1}\right) \stackrel{f}{\rightarrow}\left(X_{2}, \mathcal{U}_{2}\right)\right)=\left(X_{1}, \tau_{U_{1}}\right) \stackrel{f}{\rightarrow}\left(X_{2}, \tau_{U_{2}}\right),
$$

Likewise if $\mathbf{D}_{\pi} \mathbf{D i U}_{0}$ is a concrete category over ifD $\mathbf{D}_{\pi}$ Ditop $_{0}$ via the forgetful functor $\mathfrak{F}^{5}$ defined in Section 2.

We are now in the position to prove a similar equivalence to Lemma 3.3:

Theorem 5.2. The space $(S, \mathcal{P}(S), \pi, \mathfrak{U}) \in \mathrm{Ob}$ if $\mathbf{D}_{\pi} \mathbf{D i U}$ is $\pi$-complete if and only if the corresponding uniform space $(S, \underline{u}(\mathcal{U})) \in$ Ob Uni is complete.

Proof. Necessity. Let $(S, \mathcal{P}(S), \pi, \mathcal{U}) \in \mathrm{Ob}_{\text {ifD }}$ DiU be $\pi$-complete and take a $u(\mathcal{U})$-Cauchy filter $\mathcal{F} \subseteq \mathcal{P}(S)$. In this case, $\mathcal{F} \times \pi(\mathcal{F})$ is a complemented difilter on the texture $(S, \mathcal{P}(S))$. With the definition of cofilter $\pi(\mathcal{F})$, it is easy to verify that the difilter $\mathcal{F} \times \pi(\mathcal{F})$ is regular.

Now let us show $\mathcal{F} \times \pi(\mathcal{F})$ is $\mathcal{U}$-Cauchy:

Firstly, note that if $\mathcal{C}$ is a dicover in $\mathcal{U}$ then we can define a cover $d=\{A \cap(S \backslash B) \mid(A, B) \in \mathcal{C}\}$ in the uniformity $\underline{u}(\mathcal{U})$. Since $\mathcal{F}$ is $\underline{u}(\mathcal{U})$-Cauchy, $d \cap \mathcal{F} \neq \emptyset$ and there exists $C \in d \cap \mathcal{F}$. In this case, we have the sets $A, B$ such that $C=A \cap(S \backslash \bar{B})$ and $(A, B) \in \mathcal{C}$. Therefore $(A, B) \in \mathcal{F} \times \pi(\mathcal{F})$ and $\mathcal{F} \times \pi(\mathcal{F})$ is $U$-Cauchy difilter by $\mathcal{C} \cap(\mathcal{F} \times \pi(\mathcal{F})) \neq \emptyset$. 
As the space $(S, \mathcal{P}(S), \pi, \mathcal{U})$ is $\pi$-complete, $\pi$-complemented difilter $\mathcal{F} \times \pi(\mathcal{F})$ is diconvergent and hence there exists $s \in S$ such that $\eta(s) \times \mu(s) \subseteq \mathcal{F} \times \pi(\mathcal{F})$ due to the plainness of the texture $(S, \mathcal{P}(S))$. In view of this fact, the neighborhood filter $\mathcal{N}_{\tau}(s)$ with respect to topology $\tau$ generated by the uniformity $\underline{u}(\mathcal{U})$ will be a subfamily of $\mathcal{F}$, and we deduce that the uniform space $(S, \underline{u}(\mathcal{U}))$ is complete since the filter $\mathcal{F}$ is convergent. Sufficiency. Let the uniform space $(S, \underline{u}(\mathcal{U}))$ be complete. Take a regular $\pi$-complemented $U$-Cauchy difilter $\mathcal{F} \times \mathcal{G}$ on $(S, \mathcal{P}(S))$. Thus a filter base is defined as

$$
\mathcal{B}=\{A \cap(S \backslash B) \mid A \in \mathcal{F}, B \in \mathcal{G}\}
$$

and let $\mathcal{F}^{\prime}$ be the filter whose base is $\mathcal{B}$.

Now we will show that $\mathcal{F}^{\prime}$ is $\underline{u}(\mathcal{U})$-Cauchy: Let $d \in \underline{u}(\mathcal{U})$ be a cover. Thus the dicover $\mathcal{C}=\{(A, S \backslash B) \mid$ $\exists C \in d, A \cap B \subseteq C\} \in \mathcal{U}$ is defined and $\mathcal{C} \cap(\mathcal{F} \times \mathcal{G}) \neq \emptyset$ since $\mathcal{F} \times \mathcal{G}$ is $U$-Cauchy. Hence, there exists $(F, G) \in \mathcal{C}$ such that $F \in \mathcal{F}, G \in \mathcal{G}$ and there exists $C \in d$ such that $F \cap S \backslash G \subseteq C$. In this case, $C \in d \cap \mathcal{F}^{\prime} \neq \emptyset$ and the filter $\mathcal{F}^{\prime}$ is $\underline{u}(\mathcal{U})$-Cauchy. Next, it is convergent in $S$ by the completeness of the uniformity $\underline{u}(\mathcal{U})$. Thus, there exists an element $s \in S$ such that $\mathcal{N}_{\tau}(s) \subseteq \mathcal{F}^{\prime}$, where $\tau$ is the topology generated by the uniformity $\underline{u}(\mathcal{U})$, and so $\eta(s) \times \mu(s) \subseteq \mathcal{F} \times \mathcal{G}$ due to the equalities

$$
\eta(s)=\mathcal{N}_{\tau}(s), \quad \mu(s)=\pi(\eta(s))=\pi\left(\mathcal{N}_{\tau}(s)\right)
$$

for the complemented discrete texture $(S, \mathcal{P}(S), \pi)$. Consequently, the difilter $\mathcal{F} \times \mathcal{G}$ is diconvergent and $(S, \mathcal{P}(S), \pi, \mathcal{U}) \in \mathrm{Ob}_{\text {ifD }}$ DiU is $\pi$-complete.

Practically, note here that we have the same characterization for the subcategories $\mathbf{U n i}_{0}$ and $\mathbf{i f D}_{\pi} \mathbf{D i U}_{\mathbf{0}}$.

Remark 5.3. According to the above theorem, it is clear that the ditopological texture space $\left(S, \mathcal{P}(S), \tau_{u}, \kappa_{u}\right)$ generated by a di-uniformity $\mathcal{U}$ on $(S, \mathcal{P}(S))$ is ditopologically complete with the complementation $\pi$ if and only if the uniform space $(S, \underline{u}(\mathcal{U} \vee \pi(\mathcal{U})))$ is complete.

Therefore we can obtain the following result from Corollary 4.22 at once:

Corollary 5.4. If the space $(S, \mathcal{P}(S), \mathcal{U}) \in \mathrm{Ob}$ ifDDiU is dicomplete then the space $(S, \underline{u}(\mathcal{U} \vee \pi(\mathcal{U}))) \in \mathrm{Ob}$ Uni is complete.

For the reverse of above corollary we have a counterexample as follows:

Example 5.5. Sorgenfrey quasi-metric given in [14] as

$$
d(x, y)= \begin{cases}1 & ; x>y \\ y-x & ; x \leq y\end{cases}
$$

generates a separated quasi-uniform space $(S, Q)$ where $Q=Q_{d}$ and $S=\mathbb{Q}$ denotes the rational numbers. The space $(S, 2) \in \mathbf{O b} \mathbf{Q U}_{0}$ is an example of the bicomplete (see [10]) space which is not B-complete (see [14]), that is not Q-complete. Actually, H.P.A. Künzi mentioned that "if take any irrational number $i$ and an increasing sequence of rationals converging to $i$ as well as a decreasing sequence of rationals converging to $i$, then the filters on the set of rationals, generated in the usual way by the tails of these two sequences should yield a Cauchy filter pair (see [15]). But $i$ is not in $S$ and thus $(S, 2)$ is not B-complete."

With the above considerations, we deduce that the separated di-uniform discrete space $(S, \mathcal{P}(S), \bar{u}(Q)) \in$ Ob ifDDiU, which is an object of ifPDiU $\mathbf{U}_{0}$, is not dicomplete by Lemma 3.3 since $(S, Q) \in \mathrm{Ob}_{\mathbf{Q}} \mathbf{U}_{\mathbf{0}}$ is not Q-complete.

On the other hand, we have the (quasi-)uniformity $Q \vee Q^{-1}$ on $S$, as the coarsest uniformity contains $Q$ and the conjugate $Q^{-1}$. Moreover for the complemented di-uniformity $\bar{u}(Q) \vee \pi(\bar{u}(Q))$ we have $\underline{u}(\bar{u}(Q) \vee \pi(\bar{u}(Q)))=$ $\underline{u}(\bar{u}(Q)) \vee \underline{u}(\pi(\bar{u}(Q)))=\underline{u}(\bar{u}(Q)) \vee \underline{u}\left(\bar{u}\left(Q^{-1}\right)\right)=\underline{u}\left(\bar{u}\left(Q \vee Q^{-1}\right)=Q \mathbb{Q} \vee Q^{-1}\right.$ since the mappings $\bar{u}$, $\underline{u}$ are dual to one another and by the equality $\pi(\bar{u}(Q))=\bar{u}\left(Q^{-1}\right)$ given in [17, Proposition 3.4]. Therefore, the uniform space $\left(S, Q \vee Q^{-1}\right)$ is same with the space $(S, \underline{u}(\bar{u}(Q) \vee \pi(\bar{u}(Q))))$.

Also, it is easy to verify that the uniform space $\left(S, Q \vee Q^{-1}\right)$ is complete since it has the diagonal as an entourage and so discrete uniform space. Consequently, the space $(S, \underline{u}(\bar{u}(Q) \vee \pi(\bar{u}(Q)))) \in \mathrm{Ob}_{\mathbf{U n i}} \mathbf{i}_{0}$ which coincides with $\left(S, Q \vee Q^{-1}\right)$ is complete and the required is clear. 
Note here that the space $\left(S, \mathcal{P}(S), \pi, \bar{u}\left(\mathcal{Q} \vee Q^{-1}\right)\right) \in \mathrm{Ob}_{\mathbf{i f D}} \mathbf{D}_{\pi} \mathbf{D i U _ { 0 }}$ is $\pi$-complete by Theorem 5.2. Thus Example 5.5 is also a counterexample for the converse of Proposition 4.17, as we promised in the previous section.

Now we introduce an other required concept which will also be needed later on.

Definition 5.6. Let $(S, S, \tau, \kappa) \in \mathrm{Ob}$ ifDitop be almost plain. It is called jointly complete if the appropriate joint topology $J_{\tau \kappa}$ of $(\tau, \kappa)$ on $S_{p}$ admits a complete uniformity. ( Here, the term joint topology is defined in [pg 186, [24]])

According to the above definition we have:

Lemma 5.7. If the space $(S, \mathcal{P}(S), \tau, \kappa) \in \mathrm{Ob}$ ifDitop is ditopologically complete with the set-complementation $\pi$ then it is jointly complete.

Proof. Let $(S, \mathcal{P}(S), \tau, \kappa)$ be ditopologically complete with $\pi$. Thus there is a di-uniformity $\mathcal{U}$ such that $\tau=\tau_{\mathcal{U}}$, $\kappa=\kappa_{U}$ and the complemented di-uniform discrete texture space $(S, \mathcal{P}(S), \pi, \mathcal{U} \vee \pi(\mathcal{U}))$ is $\pi$-complete. In order to end the proof we want to find a complete uniformity $v$ such that the topology $\tau_{v}$ generated by $\mathcal{V}$ coincides with the joint topology $\mathcal{J}_{\tau \kappa}$ of $(\tau, \kappa)$ on $S_{p}$.

Clearly we have a complete uniformity $\underline{u}(\mathcal{U} \vee \pi(\mathcal{U}))$ on $S$ by Theorem 5.2 and if choose $\mathcal{V}$ as $\underline{u}(U \vee \pi(\mathcal{U}))$, then $\tau_{\mathcal{V}}=\tau_{\mathcal{U} \vee \pi(\mathcal{U})}$. In addition, the equality $\tau_{\mathcal{U} \vee \pi(\mathcal{U})}=\tau_{\mathcal{U}} \vee \tau_{\pi(\mathcal{U})}=\tau_{\mathcal{U}} \vee \pi\left(\kappa_{\mathcal{U}}\right)=\tau \vee \pi(\kappa)=\mathcal{J}_{\tau \kappa}$ is trivial due to the fact that $\left(\tau_{\pi(u),} \kappa_{\pi(u)}\right)=\left(\pi\left(\kappa_{u}\right), \pi\left(\tau_{u}\right)\right)$.

For the purpose of this section and the remainder of paper, we will define a next notion as follows:

Definition 5.8. Let $(S, \mathcal{S}, \tau, \kappa) \in \mathrm{Ob}$ ifDitop be almost plain. It is called jointly real dicompact if the joint topological space $\left(S_{p}, \partial_{\tau \kappa}\right)$ is realcompact.

Therefore the following statement arises clearly, since we are familiar with [25, Corollary 2.18 (2)]:

Proposition 5.9. If $(S, S, \tau, \kappa) \in \mathrm{Ob}$ ifDitop is real dicompact then it is jointly real dicompact.

By virtue of the definition of realcompactness for a topological space, the following proposition is obtained:

Proposition 5.10. If $(S, \mathcal{S}, \tau, \kappa) \in \mathrm{Ob}$ ifDitop is jointly real dicompact then it is jointly complete.

Proof. Let $(S, \mathcal{S}, \tau, \kappa) \in \mathrm{Ob}$ ifDitop be jointly real dicompact, so the appropriate joint topological space $\left(S_{p}, \mathcal{J}_{\tau \kappa}\right)$ is realcompact. Thus, it is easily seen that $(S, \mathcal{S}, \tau, \kappa)$ is jointly complete. Indeed, the topology $\mathcal{J}_{\tau \kappa}$ which is completely regular and Hausdorff, admits the uniformity $\mathcal{U}=\mathcal{U}_{C\left(S_{p}\right)}$ such that $\mathcal{J}_{\tau \kappa}=\tau_{\mathcal{U}}$ and $\mathcal{U}$ is complete by the following characterization given in [12, Corollary 15.14]:

"For a completely regular, Hausdorff space $(X, \tau)$, the uniform space $\left(X, \mathcal{U}_{C}\right)$ with the initial uniformity $\mathcal{U}_{C}$ generated by the ring $C=C_{\tau}(X)$ of real-valued, continuous functions on $(X, \tau)$, is (uniformly) complete if and only if the topological space $\left(X, \tau_{\mathcal{U}_{C}}\right)$ is realcompact, where $\tau=\tau_{\mathcal{U}_{C}}{ }^{\prime \prime}$.

Similar to the characterization mentioned in the end of above proof, let us recall the following equivalency given in [27, Theorem 3.8] and required for the statements which will be pointed out in the sequel.

Theorem 5.11. Let $(S, \mathcal{S}, \tau, \mathcal{\kappa}) \in \mathrm{Ob}$ ifDitop be a completely biregular, bi-T $T_{2}$ almost plain $*$-space. Then $(S, \mathcal{S}, \tau, \kappa)$ is $B$-real dicompact if and only if the di-uniform texture space $\left(S, S, v_{B}\right)$ is dicomplete. Here $B$ denotes a bigenerating sub T-lattice of $\mathrm{BA}(S)$ and $v_{B}$ denotes the initial di-uniformity generated by the T-lattice $B$, such that $\tau=\tau_{v_{B}}$, $\kappa=\kappa_{v_{B}}$. 
As a crucial aim, now we will give some statements as we promised above for a ditopological almost plain texture space $(S, S, \tau, \kappa)$, with a similar approach to the expressions given in [8].

Firstly, by the necessity part of Theorem 5.11 we have:

The space $(S, \mathcal{S}, \tau, \kappa)$ is real dicompact $(1) \Longrightarrow(S, \mathcal{S}, \tau, \kappa)$ is difilter complete (2).

Secondly, from Propositions 5.9, 5.10, we mention the following facts immediately:

The space $(S, \mathcal{S}, \tau, \kappa)$ is real dicompact $(1) \Longrightarrow(S, \mathcal{S}, \tau, \kappa)$ is jointly real dicompact $(5) \Longrightarrow(S, \mathcal{S}, \tau, \kappa)$ is jointly complete (4).

In addition, we have another requirements as follows:

The space $(S, \mathcal{S}, \tau, \kappa)$ is difilter complete $(2) \Longrightarrow(S, S, \tau, \kappa)$ is ditopologically complete $(3) \Longrightarrow(S, \mathcal{S}, \tau, \mathcal{\kappa})$ is jointly complete (4) by Corollary 4.22 and Lemma 5.7, respectively, for the texturing $\mathcal{S}=\mathcal{P}(S)$ with the complementation $\pi$.

In conclusion, the all requirements mentioned above are illustrated in the following diagram, briefly :

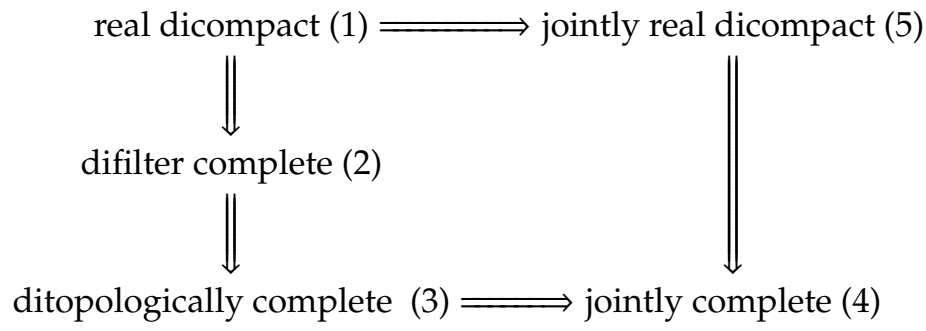

Note that the opposite directions of requirements showed in the diagram need not be true.

Actually, some of them are true for the ditopologies generated by initial di-uniformities. For, if we work with the initial di-uniformity $\mathcal{U}_{\mathrm{BA}(S)}$ generated by the $T$-lattice $\mathrm{BA}(S)$ defined on the completely biregular almost plain bi- $T_{2} *$-space $(S, \mathcal{S}, \tau, \kappa)$, it is easy to verify that the statement $(2) \Longrightarrow(1)$ is true by the sufficiency part of Theorem 5.11 .

In addition, the statements $(4) \Longrightarrow(5)$ and (3) $\Longrightarrow$ (5) are true for the ditopological spaces which are corresponding counterparts of the classical bitopological and topological spaces which have no closed discrete subspaces of Ulam-Measurable Cardinality by using [12, (Shirota) Theorem 5.20] and [12, Section 12.2].

Now, for (3) $\Longrightarrow$ (2), firstly let us prove (3) $\Longrightarrow$ (1) for the bitopological case:

If take the initial quasi-uniformity $Q$ generated by the family of pairwise continuous, real-valued functions on a bitopological space $(S, u, v)$ such that $u=\tau_{Q}, v=\tau_{Q^{-1}}$, according to the paper [9] of Brümmer and Salbany, the quasi-uniform space $(S, 2)$ is pairwise complete, that is the corresponding uniform space $\left(S, Q \vee Q^{-1}\right)$ is complete if and only if the space $\left(S, \tau_{Q}, \tau_{Q^{-1}}\right)$ is bitopologically realcompact in the sense of [9, Section 3 ], or bireal compact in the sense of [2].

As a result of the above facts, $(3) \Longrightarrow(1)$ is trivial for the ditopological discrete texture space $\left(S, \mathcal{P}(S), \tau_{\Omega},\left(\tau_{Q^{-1}}\right)^{c}\right)$ as the corresponding ditopological counterpart of the above bitopological space $\left(S, \tau_{Q}, \tau_{Q^{-1}}\right)$.

Therefore, we deduce that (3) $\Longrightarrow(2)$ is proved for the initial quasi-uniformities by using (1) $\Longrightarrow(2)$, even though it may not be true for the general case as we have seen in Example 5.5.

In addition to (3) $\Longrightarrow$ (1) which is also true for the initial di-uniformities, (3) $\Longrightarrow$ (5) will be directly true for the initial di-uniformities since (1) $\Longrightarrow(5)$ is satisfied in general.

Consequently, also note that we have a counterexample as [22, Example 4.5] for the converse of (1) $\Longrightarrow(5)$.

\section{Acknowledgement}

The author would like to thank the referees for their constructive comments, all suggestions which have helped improve the exposition and the readability of the paper. 


\section{References}

[1] J. Adámek, H. Herrlich and G. E. Strecker, Abstract and Concrete Categories, John Wiley \& Sons, Inc., 1990.

[2] L. M. Brown, Dual covering theory, confluence structures and the lattice of bicontinuous functions Ph.D. Thesis, Glasgow University, 1981.

[3] L. M. Brown, Quotients of textures and of ditopological texture spaces, Topology Proceedings 29 (2) (2005) 337-368.

[4] L. M. Brown and M. Diker, Ditopological texture spaces and intuitionistic sets, Fuzzy Sets and Systems 98 (1998), $217-224$.

[5] L. M. Brown, R. Ertürk and Ş. Dost, Ditopological texture spaces and fuzzy topology, I. Basic Concepts, Fuzzy Sets and Systems 147 (2) (2004) 171-199.

[6] L. M. Brown, R. Ertürk and Ş. Dost, Ditopological texture spaces and fuzzy topology, II. Topological considerations, Fuzzy Sets and Systems 147 (2) (2004) 201-231.

[7] L.M. Brown, R. Ertürk and Ş. Dost, Ditopological texture spaces and fuzzy topology, III. Separation Axioms, Fuzzy Sets and Systems 157 (14) (2006) 1886-1912.

[8] G.C.L. Brümmer, Two Procedures in Bitopology, in Categorical Topology, Proc.Conf.Berlin 1978, Lecture Notes in Math., Springer-Verlag, Vol.719 (1979) 35-43.

[9] G.C. L. Brümmer and S. Salbany, On the notion of real compactness for bitopological spaces, Math. Colloquium, Univ. Cape Town 11 (1977) 89-99.

[10] P. Fletcher and W. F. Lindgren, Quasi-uniform spaces, Marcel Dekker New York and Basel, 1982.

[11] G. Gierz, K. H. Hofmann, K. Keimel, J. D. Lawson, M. Mislove and D. S. Scott, A compendium of continuous lattices, SpringerVerlag, Berlin, 1980.

[12] L. Gillman and M. Jerison, Rings of Continuous Functions, D. Van Nostrand, 1960.

[13] H. Herrlich and G. E. Strecker, Category Theory, Allyn Bacon, 2nd. ed. Heldermann, Berlin, 1979.

[14] H.P. A. Künzi, C. M. Kivuvu, A double completion for an arbitrary $T_{0}$-quasi-metric space, The Journal of Logic and Algebraic Programming 76 (2008) 251-269.

[15] H.P. A. Künzi, C. M. Kivuvu, Cauchy filter pairs on quasi-uniform Spaces, Proceedings of the Workshop in Applied Topology, WIAT '10, 136-143.

[16] S. Özçağ and L. M. Brown, Di-uniform texture spaces, Applied General Topology 4 (1) (2003) 157-192.

[17] S. Özçağ and L.M. Brown, A textural view of the distinction between uniformities and quasi-uniformities, Topology and its Applications 153 (17) (2006) 3294-3307.

[18] S. Özçă̆ and Ş. Dost, A categorical view of di-uniform texture spaces, Boletin De la Sociedad de Matematica, (3) Volume 15, (2009) 63-80.

[19] S. Özçă̆, F. Yıldız and L. M. Brown, Convergence of regular difilters and the completeness of di-uniformities, Hacet. J. Math. Stat. 34 S (Doğan Çoker Memorial Issue) (2005) 53-68.

[20] İ. U. Tiryaki and L. M. Brown, Plain ditopological texture spaces, Topology and its Applications 158 (2011) 2005-2015.

[21] F. Y1ld1z, Spaces of Bicontinuous Real Difunctions and real compactness, Ph.D. Thesis (in Turkish), Hacettepe University, 2006.

[22] F. Yıldız, Connections Between Real Compactifications in Various Categories, Accepted, 2013.

[23] F. Yildız and L. M. Brown, Characterizations of real difunctions, Hacet. J. Math. Stat. 35 (2) (2006) 189-202.

[24] F. Yildiz and L. M. Brown, Categories of dicompact bi- $T_{2}$ texture spaces and a Banach-Stone theorem, Quaestiones Mathematicae 30 (2007) 167-192.

[25] F. Yıldiz and L. M. Brown, Real dicompact textures, Topology and its Applications 156 (11) (2009) 1970-1984.

[26] F. Yıldız and L. M. Brown, Real dicompactifications of ditopological texture spaces, Topology and its Applications 156 (18) (2009) 3041-3051.

[27] F. Yıldız and L. M. Brown, Dicompleteness and Real Dicompactness of Ditopological Texture Spaces, Topology and Its Applications 158 (15), (2011) 1976-1989.

[28] F. Yildiz and L.M. Brown, Extended real dicompactness and an application to Hutton spaces, Fuzzy Sets and Systems 227 (2013) 74-95. 